\title{
The 2008 Financial Crisis and Changes in Lifestyle-Related Behaviors in Italy, Greece, Spain, and Portugal: A Systematic Review
}

\author{
Monica Sane Schepisi ${ }^{1,2,+}$, Anteo Di Napoli ${ }^{1,+}$, Rosario Asciutto ${ }^{1,+}$, Simona Vecchi ${ }^{3,+}$ (D) , Concetta Mirisola ${ }^{1,+}$ \\ and Alessio Petrelli $1, *,+$ \\ 1 National Institute for Health, Migration and Poverty (INMP), 00153 Rome, Italy; \\ msaneschepisi@gmail.com (M.S.S.); anteo.dinapoli@inmp.it (A.D.N.); rosarioasciutto@gmail.com (R.A.); \\ concetta.mirisola@inmp.it (C.M.) \\ 2 Ministry of Health-General Directorate for Health Prevention, 00144 Rome, Italy \\ 3 Department of Epidemiology_Lazio Region, ASL Rome 1, 00147 Rome, Italy; s.vecchi@deplazio.it \\ * Correspondence: Alessio.petrelli@inmp.it \\ + Equally contributed.
}

check for updates

Citation: Sane Schepisi, M.; Di Napoli, A.; Asciutto, R.; Vecchi, S.; Mirisola, C.; Petrelli, A. The 2008 Financial Crisis and Changes in Lifestyle-Related Behaviors in Italy, Greece, Spain, and Portugal: A Systematic Review. Int. J. Environ. Res. Public Health 2021, 18, 8734. https://doi.org/10.3390/ijerph 18168734

Academic Editor: Paul B. Tchounwou

Received: 5 July 2021

Accepted: 14 August 2021

Published: 18 August 2021

Publisher's Note: MDPI stays neutral with regard to jurisdictional claims in published maps and institutional affiliations.

Copyright: (C) 2021 by the authors. Licensee MDPI, Basel, Switzerland. This article is an open access article distributed under the terms and conditions of the Creative Commons Attribution (CC BY) license (https:// creativecommons.org/licenses/by/ $4.0 /)$.

\begin{abstract}
Italy, Greece, Spain, and Portugal have all been strongly affected by the 2008 financial crisis, which has had a negative impact on health. We systematically evaluated the effects of the crisis on lifestyle and socioeconomic inequalities. We conducted a literature search using MEDLINE, Embase, the Cochrane Library, and health economics databases for studies reporting quantitative comparisons before and after (or during) the crisis on the following risk behaviors: alcohol consumption, smoking habit, healthy diet, physical activity, and psychotropic drugs and substance abuse, without setting any age restrictions. We selected 34 original articles published between 2011 and 2020. During/after the crisis, alcohol consumption and substance abuse decreased, while psychotropic drug use increased. We also observed a deterioration in healthy eating behavior, with a reduction in fruit and vegetable consumption. Smoking habit and physical activity showed a more complex, controversial trend. Socioeconomic inequalities were affected by the recession, and the negative effects on unhealthy lifestyle tended to be more pronounced among the disadvantaged. These results suggest the need to implement health policies and interventions aimed at monitoring risk behaviors, with special regard to disadvantaged people, and considering the potential additional impact of the COVID-19 pandemic.
\end{abstract}

Keywords: economic crisis; COVID-19; risk behaviors; lifestyles; inequalities; socioeconomic

\section{Introduction}

Before the COVID-19 pandemic, the 2008 Great Recession was the most severe crisis experienced by Europe since the Second World War, particularly in the southern European countries of Italy, Greece, Spain, and Portugal [1]. The considerable amount of literature on the topic shows that the financial crisis has had a strong impact on the health of most European populations [2]. Despite the trend towards reduced mortality, a deterioration in mental health, an increase in the number of suicides and, to a varying extent, in some non-communicable and communicable diseases, and a worsening in perceived health has been observed in most European populations [3,4]. However, an increase in mortality due to alcohol-related causes and to the consumption of drugs [5] has been observed in some countries.

Italy, Greece, Spain, and Portugal have been affected both by the direct effects of the financial crisis on the health of their populations and by the barriers to healthcare access imposed by the austerity policies introduced by governments to pay off the public debt [2]. In Greece, the austerity measures implemented to contain public spending contributed to 
an increase in forgoing health care due to economic reasons, especially among the poor, people with lower incomes, and the unemployed [6]; similar results were observed in Italy [7]. A deeper analysis by Karanikolos [2] suggests that, although recession poses risks to health, the interaction between fiscal austerity with economic shocks and weak social protection is what ultimately seems to escalate health and social crises in Europe.

The impact of the financial crisis on the health of the populations has been disproportionate. In fact, the more limited decrease in the number of deaths among the more disadvantaged social groups compared to the general population has determined a widening of mortality inequalities [8]. A recent systematic review has found an increase in socioeconomic inequalities [9]. Historical evidence supports the hypothesis that recession periods are associated with worse lifestyle, including increased alcohol consumption [10] or drug use [11], but the association between the Great Recession and worse lifestyle has not yet been systematically evaluated.

In this context, the COVID-19 pandemic began while the effects of the 2008 financial crisis were still manifesting; in addition to the dramatic impact on mortality and the direct long-term effects on the health of those who have recovered, there have also been indirect effects due to the cancellation or postponement of non-urgent assistance or interventions to decongest overwhelmed care facilities, technologies, and personnel.

The purpose of our review was to systematically evaluate the effects of the 2008 financial crisis on lifestyle and socioeconomic inequalities in Italy, Greece, Spain, and Portugal.

\section{Materials and Methods}

The review protocol was registered (CRD42019129105) in the PROSPERO open access database of systematic reviews (Available online: http:/ / www.crd.york.ac.uk/PROSPERO) (accessed on 12 August 2021). Conducting and reporting are in accordance with PRISMA guidelines. The Covidence systematic review software (Veritas Health Innovation, Melbourne, VIC, Australia. Available online: http://www.covidence.org) (accessed on 12 August 2021) was used as the reference manager tool for the phases of importation, deduplication, and selection.

\subsection{Information Sources and Search Strategy}

The literature search was performed using MEDLINE (via Ovid), Embase, The Cochrane Library and health economics databases (EconLit). Search terms for financial crisis were combined with the terms "eating behavior", "smoking habit", "alcohol consumption", "psychotropic drug use", "drug abuse", or "gambling". Supplementary Table S1 shows the full search strategy for MEDLINE. To identify additional relevant documents, the grey literature was searched for using OpenGrey and through the screening of the websites of the following referral organizations on population health and healthcare: The World Health Organization, the Organization for Economic Co-operation and Development, the European Observatory on Health Systems and Policies, the European Commission, and the European Centre for Disease Prevention and Control.

The references of included articles were also screened to identify potentially eligible articles for inclusion.

We included observational studies reporting quantitative comparisons before and after, before and during, or during and after the crisis of the following key health behaviors: diet, smoking, physical activity, alcohol consumption, and psychotropic drug use or substance abuse affecting individuals of any age. We considered studies published between January 2008 and November 2020 in English, Italian, Spanish, Portuguese, and Catalan. Multicountry studies were included when individual country data were available.

The exclusion criteria were:

1. Type of study: publications lacking primary data and/or explicit descriptions of the methods. Abstracts, editorials, correspondence, and commentaries were deemed acceptable for inclusion if they reported sufficient data;

2. Methodology: pre-post comparison missing; 
3. Data not suitable for extraction (e.g., reporting data on a group of countries);

4. Study population overlap.

Studies were selected through a three-step selection procedure based on: (1) screening of title and abstract, (2) screening of full-text article, and (3) final screening during the data extraction phase. Two independent researchers with experience in reviews and in the topic filtered and selected the references. In cases of discordance, a third researcher was consulted to determine inclusion or exclusion of the reference.

\subsection{Data Extraction, Quality Evaluation, and Synthesis of Results}

The following data were extracted from each included article: study population (number, age range, sex), population characteristics (e.g., students, household members), data source (e.g., national registry, questionnaires), study design, outcome definition, results (if available, by sex and socioeconomic status), main conclusions.

Two independent researchers judged the quality of each eligible study using a modified Newcastle-Ottawa Scale (NOS) for cross-sectional studies [12]: a study is assigned a maximum total score (stars) of nine for the following domains: selection, comparability, and outcome. We considered scores of $0-3,4-6$, and 7-9 as indications of low, medium, and high quality, respectively. Any doubt was resolved by consulting with a third reviewer. The score of the included articles are shown in the Supplementary Table S2.

The results, organized by country, report study population (number and age range) and data sources, outcome definition, results, and effects on inequalities, if reported. Given the highly heterogeneous nature of the studies, we did not attempt to conduct a metaanalysis, and we report the results narratively.

\section{Results}

Our search identified 2325 unduplicated records; after the selection process, 31 crosssectional studies [13-43] were identified, as detailed in the PRISMA flow diagram (Figure 1). The main characteristics of the eligible studies are reported in Table 1 . The articles, published between 2011 and 2020, were conducted primarily in Spain $(n=19)$; the remainder were conducted in Italy $(n=6)$, Greece $(n=6)$, and Portugal $(n=4)$. Two studies were multi-country $[19,34]$. Of the studies included, six also considered a juvenile population (age $\leq 15$ years) [21,29,33,34,41,43], while the remaining were on adolescents aged $>15$ years and on adults. The studies used validated questionnaires or administrative registries as data sources.

Regarding the outcomes considered, most of the studies concerned diet $(n=18)$ and smoking habit $(n=17)$, followed by alcohol consumption $(n=15)$ and physical activity $(n=13)$. Antidepressant/anxiolytic/antipsychotic drug use was examined by nine studies and substance abuse by six. In order to report briefly the main findings of the studies from a public health perspective, we created Table 2, which shows the variation in health behavior during or after the 2008 crisis by means a symbol for each risk behavior.

Regarding study quality, the available evidence was affected by a high risk of bias for exposure and for outcome assessment due to the study design, use of self-reported measures, and lack of adjusting for potential confounding factors. Supplementary Table S2 summarizes the NOS assessment of the included studies, which obtained scores between 2 and 7, with an average score of 4.7; four studies appeared to be of very low quality (total score 2 or 3). Confidence in ascertainment of exposure and of outcome assessment was very low in most studies. Even though most studies used a large sample of individual-level data, most data were collected by surveys that collected information using questionnaires on self-reported changes in several indicators on health-related behaviors as well as on consumption of medications. Some studies used aggregate data, which could mask individual-level effects, or self-reported questionnaires that had not been previously validated. Only six studies received a total score of $>7$, which was considered high quality. 


\subsection{The Financial Crisis and Lifestyle-Related Behaviors}

\subsubsection{The Financial Crisis and Alcohol Consumption}

Compared to the pre-crisis period, alcohol consumption decreased during or after among adults in Spain, Greece, and Italy $[16,19,20,25-29,35]$, and among young Spanish people $[13,43]$. Two studies showed a significant upward overall trend in binge drinking [20]. Conversely, two studies provided evidence of increased prevalence of moderate [18] and heavy alcohol intake after the recession [16], while a Spanish study showed no differences [17].

When stratifying by socioeconomic level, controversial results were observed. Two studies suggested a widening of socioeconomic inequalities, although this effect is due to contrasting results: one study showed that heavy alcohol consumption increased during the crisis among the least educated men [16], while another showed an increase among the most educated people [17]. Finally, a more relevant reduction in alcohol intake among the lower class was also observed elsewhere, showing a narrowing of inequalities [25].

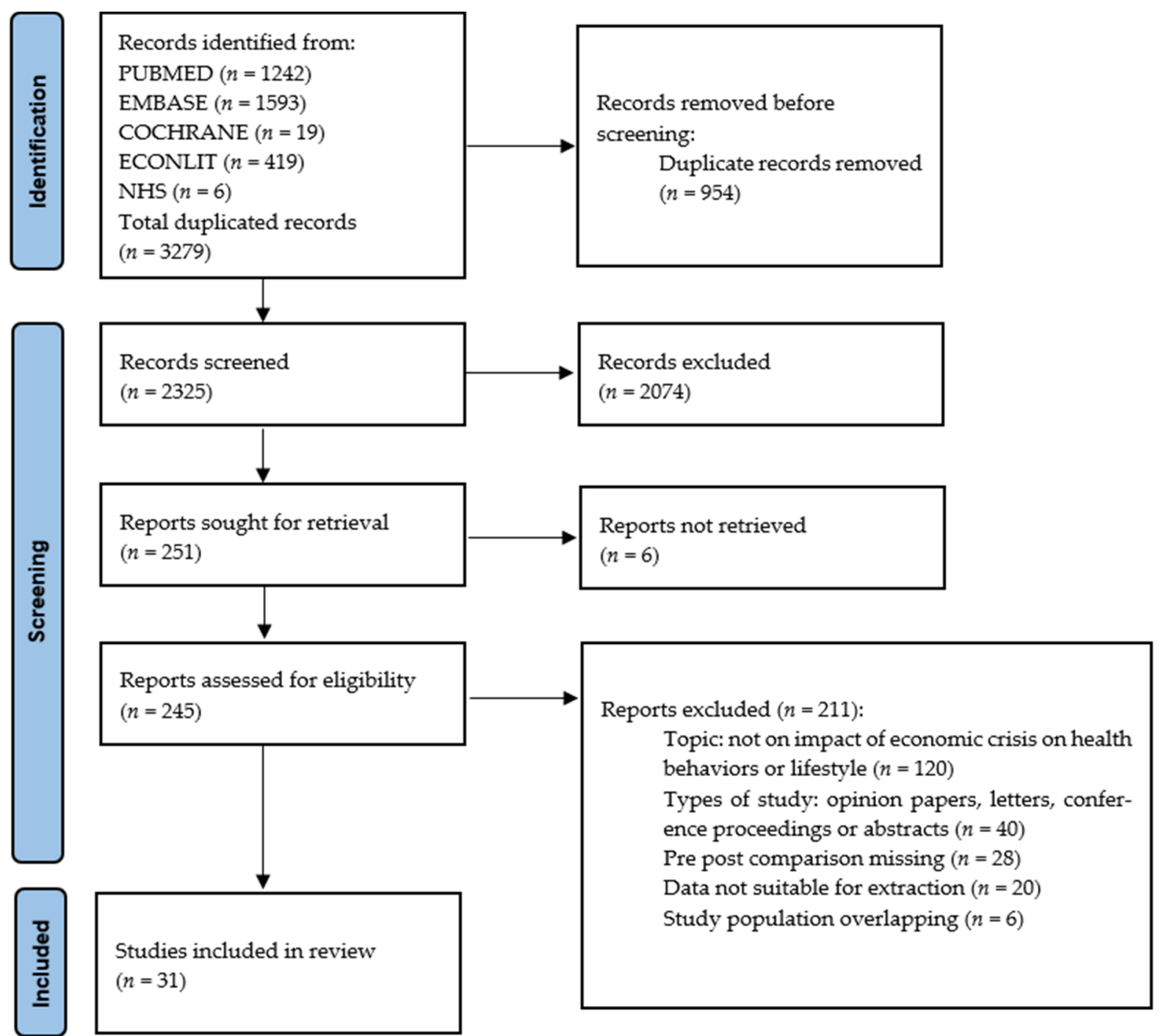

Figure 1. PRISMA flow diagram. 
Table 1. Characteristics of the included studies by country.

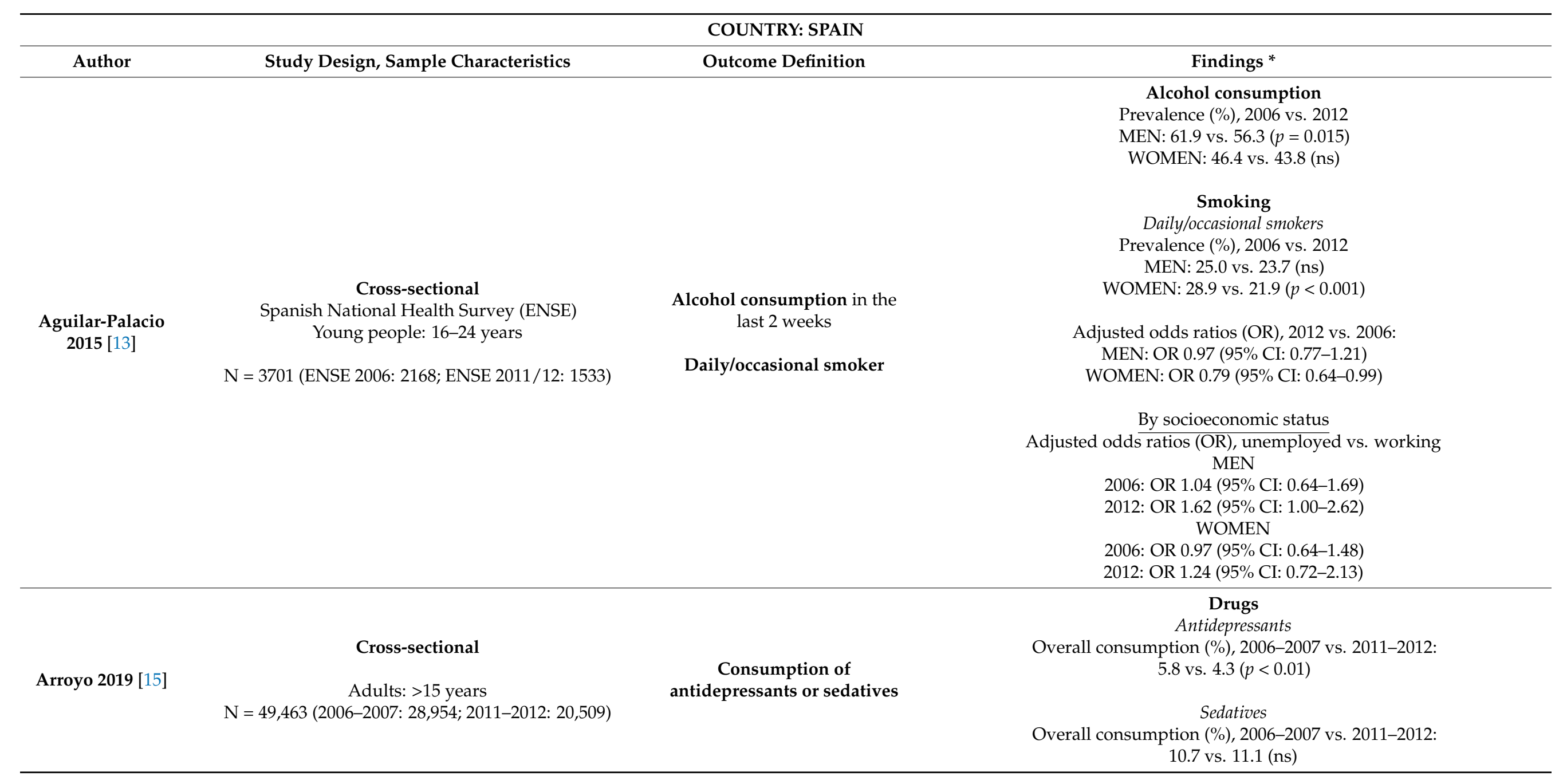


Table 1. Cont.

COUNTRY: SPAIN

Author Study Design, Sample Characteristics

\section{Cross-sectional}

Spanish National Health Survey (2001, 2003/04, 2006/07

$$
\text { and 2011/12) }
$$

Economically active adults: $25-64$ years

$$
\mathrm{N}=47,156
$$

\section{Outcome Definition}

Alcohol consumption in the last 2 weeks

Heavy alcohol consumption more than 17 Standard Basic Units of alcohol per week

\section{Smoking habits}

\section{Frequency of consumption of} food

\section{Physical activity} moderate or intense physical activity

\section{Tranquilizer or sleeping} tablet intake

at least 1 tablet in the last 2 weeks.

\section{Findings *}

\section{Alcohol}

Trend percentages (\%) of consumption, 2001-2012

Last two weeks: MEN: $-5.4(p<0.01)$; WOMEN: $-6.9(p<0.01)$

Heavy alcohol consumption: MEN: +2.0 ( $p<0.01)$; WOMEN: -0.4 (ns)

\section{By socioeconomic status}

Adjusted regression coefficients (\%) and p-value of the interaction between economic recession dummy (2001-2006/2007 vs. 2011/2012) and employment status (employed; unemployed) WOMEN

$$
\text { Last two weeks }-5.0 ;-12.4(p=0.054)
$$

Adjusted regression coefficients (\%) and $p$-value of the interaction between economic recession dummy (2001-2006/2007 vs. 2011/2012) and education level (university, high secondary, lower secondary or primary, without any

$$
\text { qualification) }
$$

$$
\text { MEN: }
$$

Heavy alcohol consumption $+0.2 ;+0.8 ;+3.1 ;+5.4(p=0.012)$

$$
\text { WOMEN: }
$$

Heavy alcohol consumption $-1.5 ;+0.1 ;+1.4 ;-0.2(p=0.012)$

\section{Smoking}

Daily or occasional smokers Trend percentages (\%), 2001-2012

MEN: $-0.8(\mathrm{~ns})$; WOMEN: $+4.4(p<0.01)$

\section{Diet}

Trend percentages (\%) of consumption, 2001-2012 Fruits: MEN: $-9.1(p<0.01)$; WOMEN: $-7.9(p<0.01)$

Vegetables: MEN: $-0.2(\mathrm{~ns})$; WOMEN: $-2.4(\mathrm{~ns})$

Legumes: MEN: $+3.4(p<0.05)$; WOMEN: $+4.3(p<0.01)$

Meat: MEN: $-9.7(p<0.01)$; WOMEN: $-10(p<0.01)$

Cold meat: MEN: $-4.7(p<0.01)$; WOMEN: $-3.7(p<0.05)$ 
Table 1. Cont.

\section{Findings *}

By socioeconomic status

Adjusted regression coefficients (\%) and $p$-value of the interaction between economic recession dummy (2001-2006/2007 vs. 2011/2012) and employment status (employed; unemployed)

$$
\text { MEN: }
$$

Vegetables daily: $+9.0 ; 6.3(p=0.004)$

Fruits daily: $-7.4 ;-12.1(p=0.041)$

Legumes (3 times or more per week): $+3.8 ;-7.4(p=0.041)$

Fish (3 times or more per week): $+1.2 ;-6.4(p=0.055)$

$$
\text { WOMEN }
$$

Vegetables daily: $-1.7 ;-4.3(p=0.065)$

Adjusted regression coefficients (\%) and $p$-value of the interaction between economic recession dummy (2001-2006/2007 vs. 2011/2012) and education level (university, high secondary, lower secondary or primary, without any qualification)

$$
\text { MEN: }
$$

Fruits daily: $-4.5 ;-6.1 ;-11.4 ;-21.8(p=0.06)$

Sweet food (3 times or more per week): $-1.7 ;+2.5 ;+3.1 ;-16.9(p=0.067)$ WOMEN:

Vegetables daily: $-1.3 ;+3.4 ;-3.0 ;-27.1(p=0.004)$

\section{Physical activity}

Moderate or intense

Trend percentages (\%), 2001-2012

MEN: +3.2 $(p<0.05)$; WOMEN: +1.4 (ns)

By socioeconomic status

Adjusted regression coefficients (\%) and $p$-value of the interaction between economic recession dummy (2001-2006/2007 vs. 2011/2012) and education level (university, high secondary, lower secondary or primary, without any qualification

WOMEN:

$+6.5 ;+4.6 ;-1.6 ;-0.7(p=0.014)$ 
Table 1. Cont.

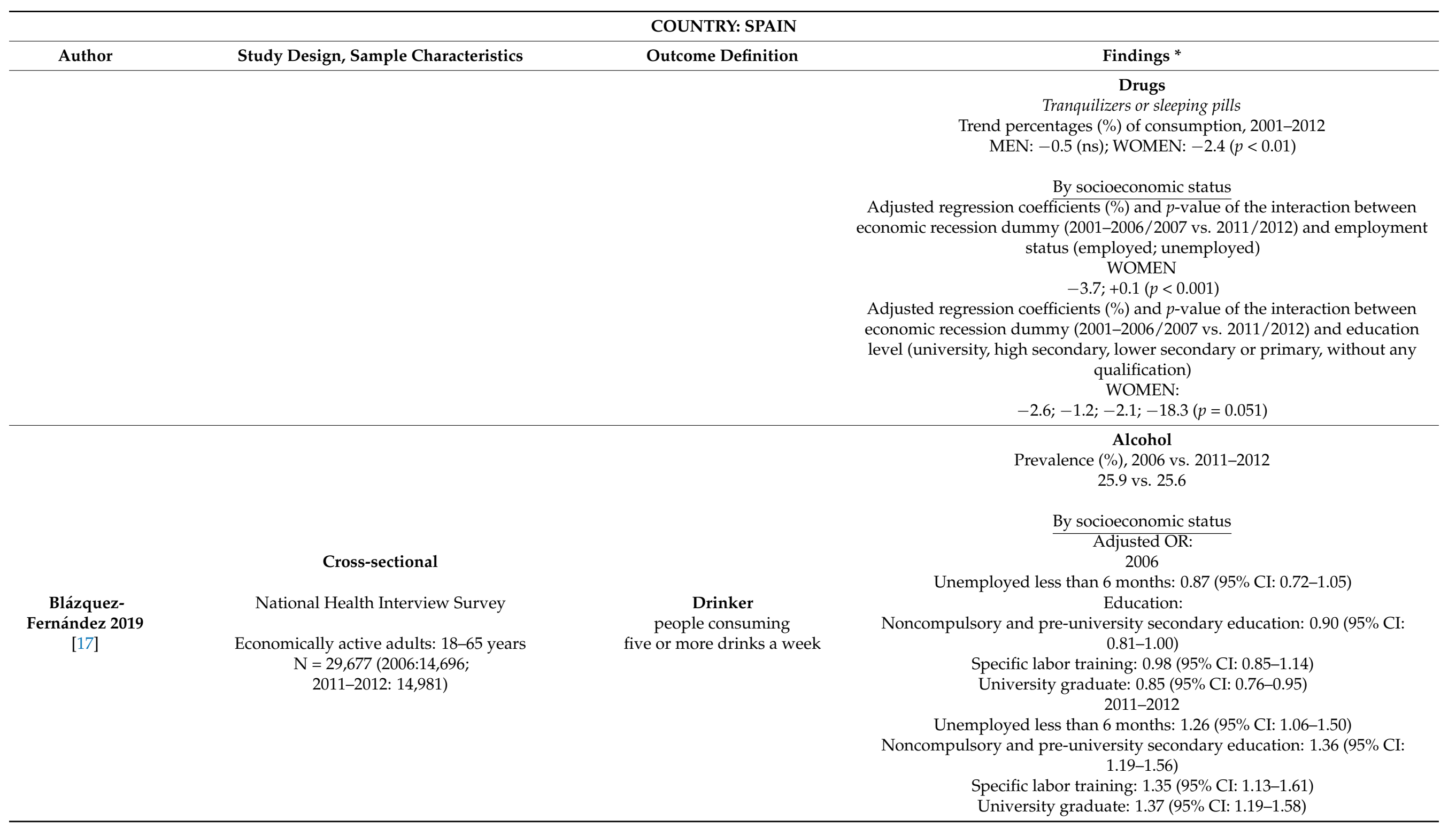


Table 1. Cont.

\section{COUNTRY: SPAIN}

Author

Colell 2015 [20]

Economically active adults: 50-64 years $\mathrm{N}=62,440$
Outcome Definition

Daily average of alcohol

intake in the last 30 days

(grams of pure ethanol)

\section{Heavy drinkers}

above $\geq 40 \mathrm{~g}$ for men and $\geq$

$24 \mathrm{~g}$ for women

\section{Binge drinking}

5 or more drinks on a single drinking occasion (within $2 \mathrm{~h}$ )

at least once in the previous

month in editions 2005 and 2007.

Editions 2009 and 2011: 5 or more drinks for men and four or more for women

\section{Hypnotics/sedatives}

Sporadic users: use from 1 to 9 days in the last 30 days

Heavy users: use from 10 to 30 days in the last 30 days

\section{Findings}

\section{Alcohol}

Daily average of alcohol intake (g/day), 2005-2007 vs. 2009-2011

MEN: 16.9 vs. $15.1(p<0.001)$

WOMEN: 7.7 vs. $7.1(p=0.002)$

Heavy drinking

Prevalence (\%), 2005-2007 vs. 2009-2011

MEN: 6.9 vs. $5.2(p<0.001)$

WOMEN: 3.3 vs. $2.8(p=0.013)$

Adjusted prevalence ratio (PR) (ref pre-crisis): MEN: 0.73 (95\% CI: 0.67-0.79)

WOMEN: 0.86 (95\% CI: 0.75-0.99)

Binge drinking

Prevalence (\%), 2005-2007 vs. 2009-2011

MEN: 19.3 vs. $22.0(p<0.001)$

WOMEN: 7.1 vs. $10.1(p<0.001)$

Adjusted prevalence ratio (PR) (ref pre-crisis):

MEN: 1.17 (95\% CI: 1.12-1.22)

WOMEN: 1.62 (95\% CI: 1.49-1.76)

\section{Drugs}

Hypnotics/sedatives sporadic users

Prevalence (\%), 2005-2007 vs. 2009-2011

MEN: 1.2 vs. $1.6(p=0.005)$

WOMEN: 2.1 vs. 2.4 (ns)

Hypnotics/sedatives heavy users

Prevalence (\%), 2005-2007 vs. 2009-2011

MEN: 1.8 vs. $2.1(p=0.005)$

WOMEN: 3.7 vs. $5.4(p<0.001)$

Prevalence ratio (PR) (ref pre-crisis):

MEN: 1.19 (95\% CI: 0.99-1.42)

WOMEN: 1.32 (95\% CI: 1.17-1.49) 
Table 1. Cont.

\section{COUNTRY: SPAIN}

Author

Study Design, Sample Characteristics

Outcome Definition

Interaction (RRR) between activity (unemployed vs. employed) and period

(2009-2011 vs. 2005-2007)

MEN: 0.69 (95\% CI: 0.49-0.97)

Substance abuse

Cannabis sporadic users

Prevalence (\%), 2005-2007 vs. 2009-2011

MEN: 5.6 vs. 5.2 (p ns)

WOMEN: 3.1 vs. $2.4(p<0.001)$

Adjusted prevalence ratio (PR) (ref pre-crisis)

MEN: 0.90 (95\% CI: 0.81-1.01)

WOMEN: 0.77 (95\% CI: 0.64-0.91)

By socioeconomic status

Interaction (RRR) between activity (unemployed vs. employed) and period

(2009-2011 vs. 2005-2007)

MEN: 1.40 (95\% CI: 1.10-1.77)

WOMEN: 1.68 (95\% CI: 1.17-2.41)

Cannabis heavy users

Prevalence (\%), 2005-2007 vs. 2009-2011

MEN: 6.0 vs. 5.7 (ns)

WOMEN: 2.0 vs. 1.9 (ns)

\section{Diet}

Trend 2006-2011

Fruits (daily): falling

\section{Cross-sectional}

Diaz-Mendez 2019 [22]
Adults $>16$ years

$N=50,485$ (2006: 29,478; 2011-2012: 21,007)
Frequency of consumption of

food
Meat ( 3 or more times a week): rising

Eggs ( 3 or more times a week): falling

Fish (3 or more times a week): falling

Pasta-rice-potatoes (daily): falling

Bread (daily): remaining within guidelines.

Vegetables (daily): falling

Pulses (once or twice a week): rising

Processed meats (occasionally/seldom or never): continuing 
Table 1. Cont.

\section{COUNTRY: SPAIN}

Author Study Design, Sample Characteristics

Outcome Definition

Alcohol use during the last 2 weeks

\section{Cross-sectional}

\section{Garcia-Mayor} 2019 [25]

Spanish National Health Survey (SNHS)

Adults $18-64$ years

$N=51,370$ (2006: 28,478; 2012: 21,007; 2017: 23,089)

\section{Tobacco use}

Fruit vegetable, pastries and/or sweets, sweetened beverages

daily intake (yes or no)
Findings *

Dairy (daily): falling

Sweets (occasionally/seldom or never): falling Soft drinks (occasionally/seldom or never): continuing

\section{Alcohol}

Differences in prevalence, 2012 vs. 2006, 2017 vs. 2006

MEN: $-4.7 \%(p<0.001),-8.7 \%(p<0.001)$

WOMEN: $-3.3 \%(p<0.001),-5.8 \%(p<0.001)$ By socioeconomic status

High $(-2.8 \%,-4.4 \%)$ Middle $(-1.2 \%,-4.0 \%)$ Low $(-0.8 \%,-7.2 \%)$

\section{Smoking}

Differences in prevalence, 2012 vs. 2006, 2017 vs. 2006 MEN: $-3.5 \%(p<0.001),-7.7 \%(p<0.001)$ WOMEN: $+0.3 \%$ (ns), $-1.6 \%$ (ns)

By socioeconomic status

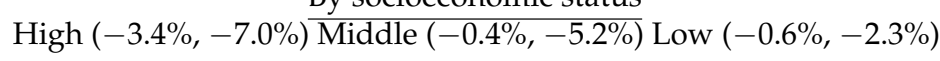

\section{Diet}

Fruit consumption

Differences in prevalence, 2012 vs. 2006, 2017 vs. 2006 MEN: $-3.9 \%(p<0.001),-4.6 \%(p<0.001)$

WOMEN: $-7.2 \%(p<0.001),-4.9 \%(p<0.001)$ By socioeconomic status

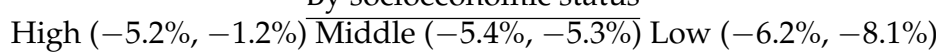
Vegetable consumption

Differences in prevalence, 2012 vs. 2006, 2017 vs. 2006 MEN: $+4.8 \%(p<0.001),-2.1 \%(p=0.005)$

WOMEN: $+3.5 \%(p<0.001),-0.7 \%$ (ns)

By socioeconomic status

High $(+4.6 \%,+2.3 \%)$ Middle $(+5.2 \%,-1.0 \%)$ Low $(+2.1 \%,-3.8 \%)$ Sweets consumption

Differences in prevalence, 2012 vs. 2006, 2017 vs. 2006

MEN: $-4.4 \%(p<0.001),-8.8 \%(p<0.001)$

WOMEN: $-6.7 \%(p<0.001),-9.3 \%(p<0.001)$ By socioeconomic status

High $(-3.5 \%,-8.9 \%)$ Middle $(-7.7 \%,-9.9 \%)$ Low $(-5.4 \%,-9.2 \%)$ 
Table 1. Cont.

COUNTRY: SPAIN

Author Study Design, Sample Characteristics

Outcome Definition

Alcohol consumption In the last month (EDADES) Habitual (ENS)

\section{Cross-sectional}

Marquez-

Calderon 2014

[27]
Enquesta Domiciliaria sobre Alcohol y Droga en Espana (EDADES) Adults 15-64 years

Enquesta Nacional de Salud de Espana (ENS)
Smoking habits

In the last month (EDADES)

Daily (ENS)

\section{Drugs use}

In the last month: sedatives, tranquilizer, hypnotics

$$
\text { (EDADES) }
$$

Last 2 weeks: antidepressants

(ENS)
Findings *

Sweetened beverages

Differences in prevalence, 2012 vs. 2006, 2017 vs. 2006 MEN: $-4.7 \%(p<0.001),-3.5 \%(p<0.001)$

WOMEN: $-8.9 \%(p<0.001),-5.0 \%(p<0.001)$

By socioeconomic status

High $(-2.2 \%,-7.1 \%)$ Middle $(-4.1 \%,-5.4 \%)$ Low $(-5.2 \%,-8.1 \%)$

Physical activity

Differences in prevalence, 2012 vs. 2006, 2017 vs. 2006

MEN: $+0.7 \%(\mathrm{p}: \mathrm{ns}),+4.9 \%(p<0.001)$

WOMEN: $-4.7 \%(p<0.001),+3.3 \%(p<0.001)$

By socioeconomic status

High $(-0.1 \%,+7.6 \%)$ Middle $(-0.3 \%,+6.3 \%)$ Low $(-2.1 \%,+3.6 \%)$

\section{Alcohol}

Prevalence (\%), 2005 vs. 2011 (EDADES) and 2006 vs. 2012 (ENS) EDADES: 64.6 vs. 62.3 ; ENS: 48.4 vs. 38.3

\section{Smoking}

Prevalence (\%), 2005 vs. 2011 (EDADES) and 2006 vs. 2012 (ENS) EDADES: 38.4 vs. 37.6; ENS: 26.4 vs. 24.0

\section{Drugs}

Prevalence (\%), 2005 vs. 2011

Sedatives (EDADES): 3.7 vs. 8.3 ;

Tranquilizers (EDADES): 2.7 vs. 6.9;

Hypnotics (EDADES): 2.0 vs. 3.4;

Antidepressants (ENS): 8.5 vs. 7.0

Physical activity

Sedentary lifestyle

Prevalence (\%), 2006 vs. 2012: $39.4 \%$ vs. $41.3 \%$ 
Table 1. Cont.

\section{COUNTRY: SPAIN}

Author

Study Design, Sample Characteristics

\section{Cross-sectional}

Martin Bassols 2016 [28]
People aged 15-64 years N = 92,102 (2005: 27,400; 2007: 23,276; 2009: 19,713; 2011: 21,713)

Outcome Definition

\section{Alcohol consumption}

\section{Smoking habits}

Substance abuse marijuana and hard drugs such as crack, cocaine, heroin ecstasy, hallucinogens, inhalants, and amphetamines in the last 12 months, last 30 days, every day in last 30 days

\section{Findings *}

\section{Substance abuse}

Prevalence (\%), 2005 vs. 2011

Cannabis: 8.7 vs. 7.0

Ecstasy: 0.6 vs. 0.3

Hallucinogens: 0.2 vs. 0.2

Amphetamines: 0.4 vs. 0.3

Cocaine powder: 1.6 vs. 1.1

Cocaine base: 0.1 vs. 0.1

Heroin: 0.1 vs. 0.1

\section{Alcohol}

Mean (\%) 2005, 2007, 2009, 2011

Alcohol in last 12 months: 76.63, 72.92, 79,17, 77.08

Drunk in last 12 months: 22.01, 19.60, 27.16, 22.44

Alcohol in last 30 days: $63.24,58.98,62.48,60.58$

Alcohol every day in last 30 days: 12.05, 9.27, 9.31, 8.68

By socioeconomic status

Probability (\%) of consuming alcohol given a $10 \%$ increase in the provincial unemployment rate

Alcohol in the past 12 months: $-3.4(p<0.1)$

Not consuming any alcohol: $+3(p<0.05)$

Consuming alcohol fewer than 20 days in the last year: $1.1(p<0.05)$

Consuming alcohol between 20 and 29 days during the last year: -0 . $(p<0.05)$

Consuming alcohol between 30 and 150 during the last year: -1.7 $(p<0.05)$

Consuming alcohol more than 150 days during the last year: -2.3

$(p<0.05)$

Smoking

Mean (\%) 2005, 2007, 2009, 2011

Smoked in last 12 months: 31.81, 29.21, 31.78, 31.19 
Table 1. Cont.

Findings *

By socioeconomic status

Probability (\%) of smoking tobacco given a $10 \%$ increase in the provincial unemployment rate

Smoked daily during the last 12 months: $+3(p<0.01)$

\section{Substance abuse}

Drug consumption in last 12 months

Mean (\%) 2005, 2007, 2009, 2011

Marijuana: $12.79,10.56,13.21,11.38$

Hard drugs: $4.03,3.73,3.99,3.47$

Cocaine: $3.31,2.96,3.09,2.81$

Ecstasy: 1.46, 1.17, 1.19, 0.91

Drug consumption in last 30 days

Mean (\%) 2005, 2007, 2009, 2011

Marijuana: 9.79, 7.42, 9.40, 8.26

Hard drugs: $2.21,2.00,1.93,1.46$

Cocaine: $1.76,1.64,1.43,1.33$

Ecstasy: $0.62,0.41,0.50,0.33$

By socioeconomic status

Probability of using drugs in last 12 months given a $10 \%$ increase in the provincial unemployment rate

Marijuana: $+3.1(p<0.01)$

Hard drugs: +0.9 (ns)

Cocaine: $+1.2(p<0.01)$

Ecstasy: $-0.4(\mathrm{~ns})$

Probability of using drugs in last 30 days given a $10 \%$ increase in the provincial unemployment rate

Marijuana: $+2.4(p<0.01)$

Hard drugs: $+0.7(\mathrm{~ns})$

Cocaine: $+0.9(p<0.1)$

Ecstasy: $-0.2(\mathrm{~ns})$ 
Table 1. Cont.

\begin{tabular}{|c|c|c|c|}
\hline & & COUNTRY: SPAIN & \\
\hline Author & Study Design, Sample Characteristics & Outcome Definition & Findings * \\
\hline $\begin{array}{l}\text { Moreno Lostao } \\
\quad 2019[30]\end{array}$ & $\begin{array}{l}\text { Spanish National Health Survey. } \\
\text { People aged 15-74 years }\end{array}$ & $\begin{array}{c}\text { Tobacco consumption } \\
\text { daily and occasional smokers } \\
\text { Physical inactivity } \\
\text { no physical exercise and } \\
\text { leisure time spent in sedentary } \\
\text { habits }\end{array}$ & 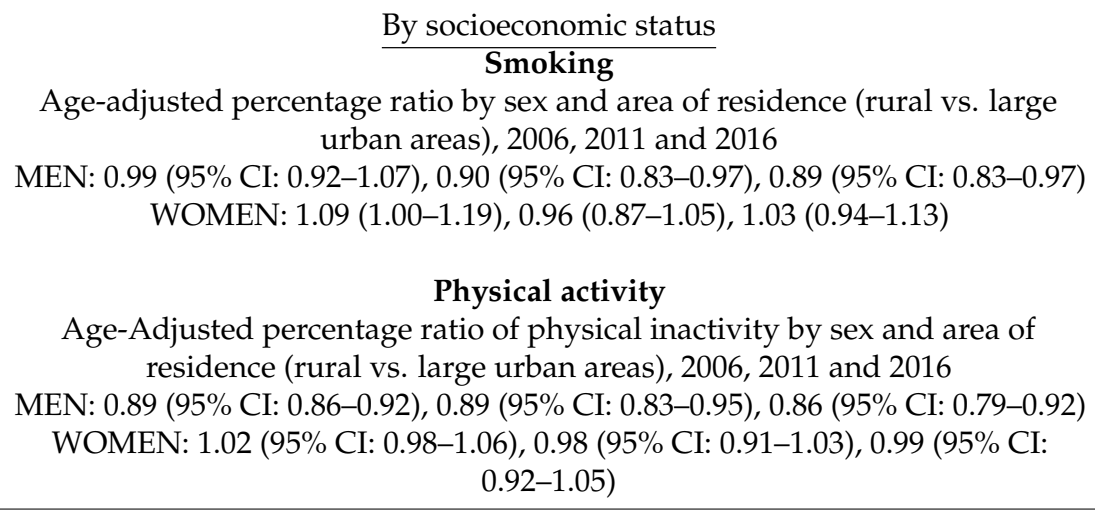 \\
\hline $\begin{array}{l}\text { Perez-Romero } \\
2016[31]\end{array}$ & $\begin{array}{c}\text { Cross sectional } \\
\text { Spanish National Health Survey } \\
\text { Adults aged 18-64 years } \\
\mathrm{N}=30,817(2006-2007: 18,202 ; 2011-2012: 12,615)\end{array}$ & $\begin{array}{l}\text { Drugs consumption } \\
\text { hypnotics and anxiolytics in } \\
\text { the last } 2 \text { weeks }\end{array}$ & $\begin{array}{c}\text { Drugs } \\
\text { Adjusted odds ratios (OR), 2011-2012 vs. 2006-2007 } \\
\text { MEN: OR 2.3 (95\% CI: 1.8-2.8) } \\
\text { WOMEN: OR 1.7 (95\% CI: } 1.4-1.9)\end{array}$ \\
\hline \multirow{3}{*}{ Rajmil 2013 [33] } & & Junk food consumption & $\begin{array}{c}\text { Diet } \\
\text { Junk food consumption } \\
\text { Prevalence (\%), } 2006 \text { vs. } 2010-2012 \\
50.24 \text { (95\% CI: 49.74-50.74) vs. } 52.34 \text { (95\% CI: } 51.92-52.76)\end{array}$ \\
\hline & $\begin{array}{l}\text { Cross-sectional } \\
\text { Children <15 years old enrolled in Catalan Health Survey } \\
\text { (ESCA) } \\
\mathrm{N}=4167 \text { (2006: 2200; first wave 2010-2012: 1967) }\end{array}$ & $\begin{array}{l}\text { Having breakfast at home } \\
\text { never vs. at least once per } \\
\text { week } \\
\text { Physical Activity }\end{array}$ & 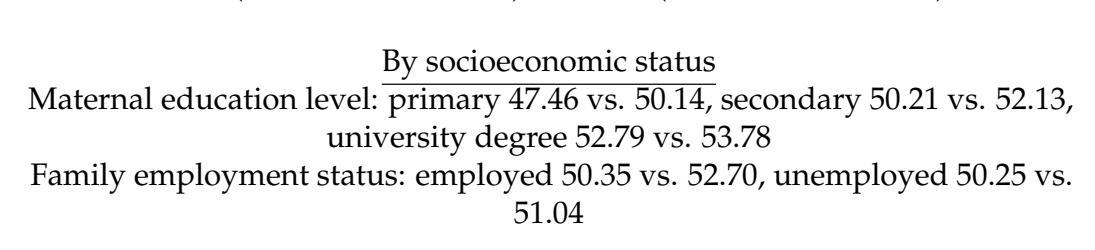 \\
\hline & & Time spent on screen & $\begin{array}{l}\text { Never having breakfast } \\
\text { Prevalence (\%), } 2006 \text { vs. } 2010-2012 \text { : } \\
4.9(95 \% \text { CI: } 3.8-6.0) \text { vs. } 5.4(4.8-6.7)\end{array}$ \\
\hline
\end{tabular}


Table 1. Cont.

\section{Findings *}

\section{By socioeconomic status}

Maternal education level: primary 5.9 vs. 7.7 , secondary 5.1 vs. 6.4 , university degree 3.5 vs. 2.7 )

Family employment status: employed 4.3 vs. 5.6, unemployed 8.8 vs. 4.4

Physical activity

Prevalence (\%), 2006 vs. $2010-2012$

50.14 (95\% CI: $49.52-50.76)$ vs. 48.23 (95\% CI: 47.59-48.87)

By socioeconomic status

Maternal education level: primary 47.46 vs. 50.14 , secondary 50.21 vs. 52.13 ,

university degree 52.79 vs. 53.86

Family employment status: employed 50.35 vs. 52.70 , unemployed 50.25 vs. 51.04

Time (hours/day) spent on screen

Mean, 2006 vs. 2010-2012

2.03 (95\% CI: $1.98-2.07)$ vs. 1.41 (95\% CI: $1.35-1.47)$

By socioeconomic status

Maternal education level: primary 2.16 vs. 1.73 , secondary 2.08 vs. 1.53 , university degree 1.77 vs. 1.07

Family employment status: employed 4.3 vs. 5.6, unemployed 8.8 vs. 4.4

$$
\text { Alcohol }
$$

Annual percentage change (APC) in different time intervals:

$-0.1(2004-2006)(\mathrm{ns})$

$-2.3(2008-2010)(p=0.024)$

$-0.2(2011-2013)(\mathrm{ns})$

$+2.1(2014-2016)(p=0.059)$ 
Table 1. Cont.

\section{COUNTRY: SPAIN}

Author Study Design, Sample Characteristics

Outcome Definition

\section{Alcohol consumption}

Tobacco consumption

number of cigarettes

sold per inhabitant aged $\geq 15$

$$
\text { years; }
$$

Regidor 2019 [35]

\section{Cross-sectional}

Data taken from different sources

\section{Fruit and vegetable intake}

measured by purchase

\section{Physical activity}

gone to a gym in the last 30

days, in population aged $\geq 15$

$$
\text { years }
$$

Findings *

\section{Smoking}

Tobacco smoking

Annual percentage change (APC) in different time intervals

$-1.9(2004-2006)(\mathrm{ns})$

$-8.3(2008-2010)(p<0.001)$

$-13.5(2011-2013)(p<0.001)$

$-1.1(2014-2016)(\mathrm{ns})$

\section{Diet}

Fruit and vegetable consumption:

Annual percentage change (APC) in different time intervals:

$$
-0.1(2004-2006)(\mathrm{ns})
$$

$2.1(2008-2010)(p<0.001)$

$1.2(2011-2013)(p=0.026)$

$-1.9(2014-2016)(p=0.003)$

Away-from-home dinners

Annual percentage change (APC) in different time intervals:

$$
-0.2(2004-2006)(\mathrm{ns})
$$

$-3.3(2008-2010)(p<0.001)$

$-1.6(2011-2013)(p=0.009)$

$3.2(2014-2016)(p<0.001)$

\section{Physical activity}

Going to a gym

Annual percentage change (APC) in different time intervals

$0.2(2004-2006)(\mathrm{ns})$

$4.4(2008-2010)(p=0.001)$

$1.6(2011-2013)(\mathrm{ns})$

$7.1(2014-2016)(p<0.001)$ 
Table 1. Cont.

COUNTRY: SPAIN

Author Study Design, Sample Characteristics

\section{Cross-sectional}

Spijker 2018 [39]

Catalan Health Survey (ESCA)

Adults aged $>50$ years

$\mathrm{N}=16,593$ (2006: 6667; 2010-2012: 4458; 2013-2015: 5469)

\section{Sedentary life}

Findings *

Physical activity

Sedentary life

Prevalence (\%), 2006, 2010-2012, 2013-2015

$$
\text { MEN: }
$$

50-64 years old: $20.8,18.3,24.4$

65+: $37.4,28.0,34.6$

WOMEN:

50-64 years old: $18.9,16.0,22.5$

$65+: 41.7,33.4,41.9$

By socioeconomic status

Occupational status (employed, unemployed)

Prevalence (\%)

2006: $0.26,0.31$

2010-2012: $0.23,0.26$

2013-2015: 0.28, 0.34

\section{Smoking}

Prevalence (\%), 2003-2004 vs. 2011-2012

Couple mothers: 35.4 vs. 29.5

Lone mothers household heads: 42.7 vs. 39.1

Lone mothers-non household heads: 78.1 vs. 42.3

Trujillo-Aleman

2019 [40]

\section{Cross-sectional}

N = 5919 mothers (2003-2004: 2951; 2011-2012: 2698)
Smoking habits

daily and not daily smokers
By socioeconomic status

Social class (non-manual, manual)

Lone mothers household heads vs. couple mothers

Adjusted prevalence ratios, 2003-2004 2011-2012

Non-manual: 1.26 (95\% CI: $1.00-1.59) 1.23$ (95\% CI: 0.95-1.58)

p interaction ns

Manual: 1.30 (95\% CI: 1.08-1.57) 1.34 (95\% CI: 1.08-1.66) 
Table 1. Cont.

\begin{tabular}{|c|c|c|c|}
\hline & & COUNTRY: SPAIN & \\
\hline Author & Study Design, Sample Characteristics & Outcome Definition & Findings * \\
\hline $\begin{array}{l}\text { Zapata Moya } \\
\quad 2020[42]\end{array}$ & $\begin{array}{l}\text { Cross-sectional } \\
\text { People aged }>=18 \\
\quad \mathrm{~N}=5679\end{array}$ & $\begin{array}{l}\text { Anxiolytics and/or } \\
\text { antidepressant consumption } \\
\text { before } 2008 \text { and in the last two } \\
\text { weeks before the interview } \\
\text { (2015) }\end{array}$ & $\begin{array}{c}\text { Drugs } \\
\text { Adjusted OR: } 2015 \text { vs. } 2008 \\
1.51 \text { (95\% CI: } 1.05-2.42) \\
\text { By socioeconomic status } \\
\text { Interaction between crisis impact on family SES and period (2015 vs. 2008) } \\
\text { Adjusted OR: } 2.18 \text { (95\% CI: } 1.48-3.16)\end{array}$ \\
\hline \multirow[t]{2}{*}{ Zozaya 2020 [43] } & \multirow[b]{2}{*}{$\begin{array}{l}\text { Children and adolescents aged 9-21 years } \\
\qquad N=77,651\end{array}$} & $\begin{array}{l}\text { Alcohol consumption } \\
\text { drinking any alcoholic } \\
\text { beverage at least } \\
\text { every week }\end{array}$ & $\begin{array}{c}\text { Alcohol } \\
\text { Prevalence (\%), 2002, 2006, 2010, } 2014 \\
\text { 17.57, 18.29, 16.09, 7.11 }\end{array}$ \\
\hline & & $\begin{array}{c}\text { Smoking habit } \\
\text { frequent or occasional } \\
\text { smoking during the last year }\end{array}$ & $\begin{array}{c}\text { Smoking } \\
\text { Prevalence }(\%) \text { 2002, 2006, 2010, } 2014 \\
24.92,16.10,17.00,10.00\end{array}$ \\
\hline & & COUNTRY: ITALY & \\
\hline
\end{tabular}

\section{Alcohol}

Mean grams/day of alcohol intake, 2005-2006 vs. 2007-2010 16.0 (SD: 21.8) vs. 16.9 (SD: 23.2) $(p<0.01)$

\section{Cross-sectional}

Moli-sani study

Adults aged $>35$ years

$$
\mathrm{N}=21,001
$$

\section{Alcohol intake}

Adherence to Mediterranean diet (MD) measured through the Italian Mediterranean Index score

\section{Diet}

$\%$ of high adherence to MD, 2005-2006 vs. 2007-2010 31.3 vs. $18.3(p<0.01)$

By socioeconomic status

Prevalence ratios (PR), 2005-2006 and 2007-2010

High Wealth Index score vs. Low Wealth Index score: 1.05 (95\% CI: 0.94-1.16) 1.31 (95\% CI: 1.18-1.46) $>13$ years of education vs. $<=8$ years of education: 1.16 (95\% CI: 1.04-1.31) 1.32 (95\% CI: 1.17-1.50) Manual non-manual job vs: 0.97 (95\% CI: 0.82-1.15) 0.67 (95\% CI: 0.57-0.79) 
Table 1. Cont.

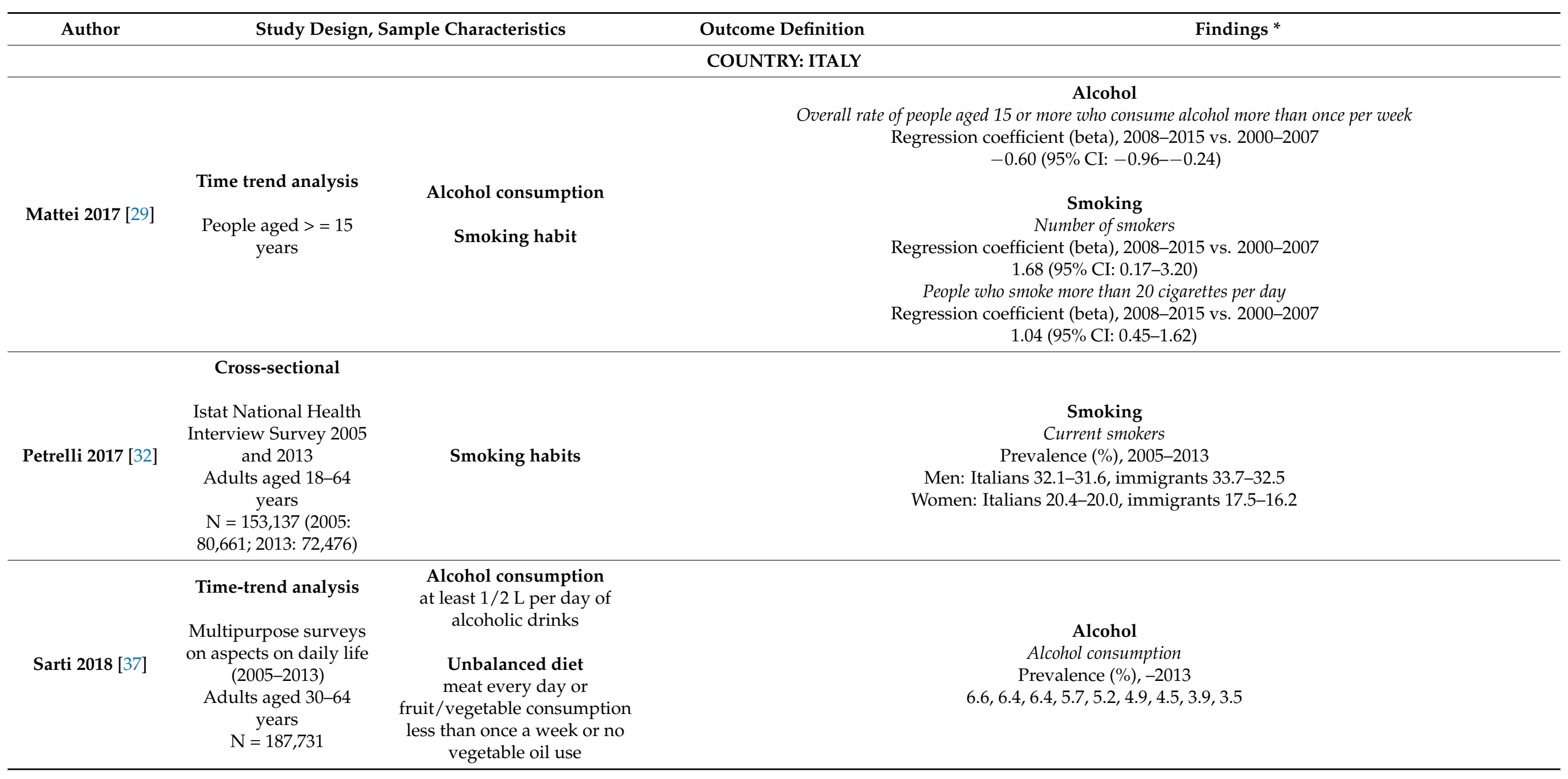


Table 1. Cont.

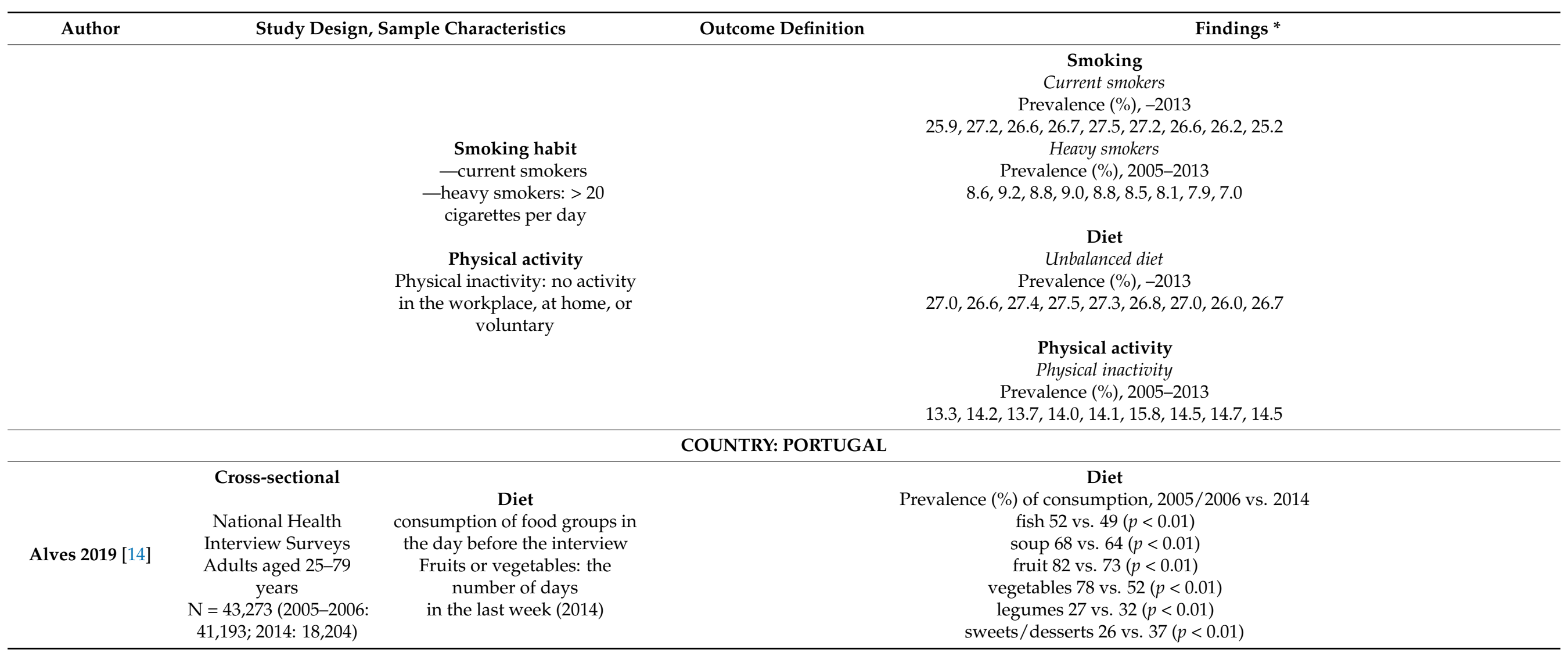


Table 1. Cont.

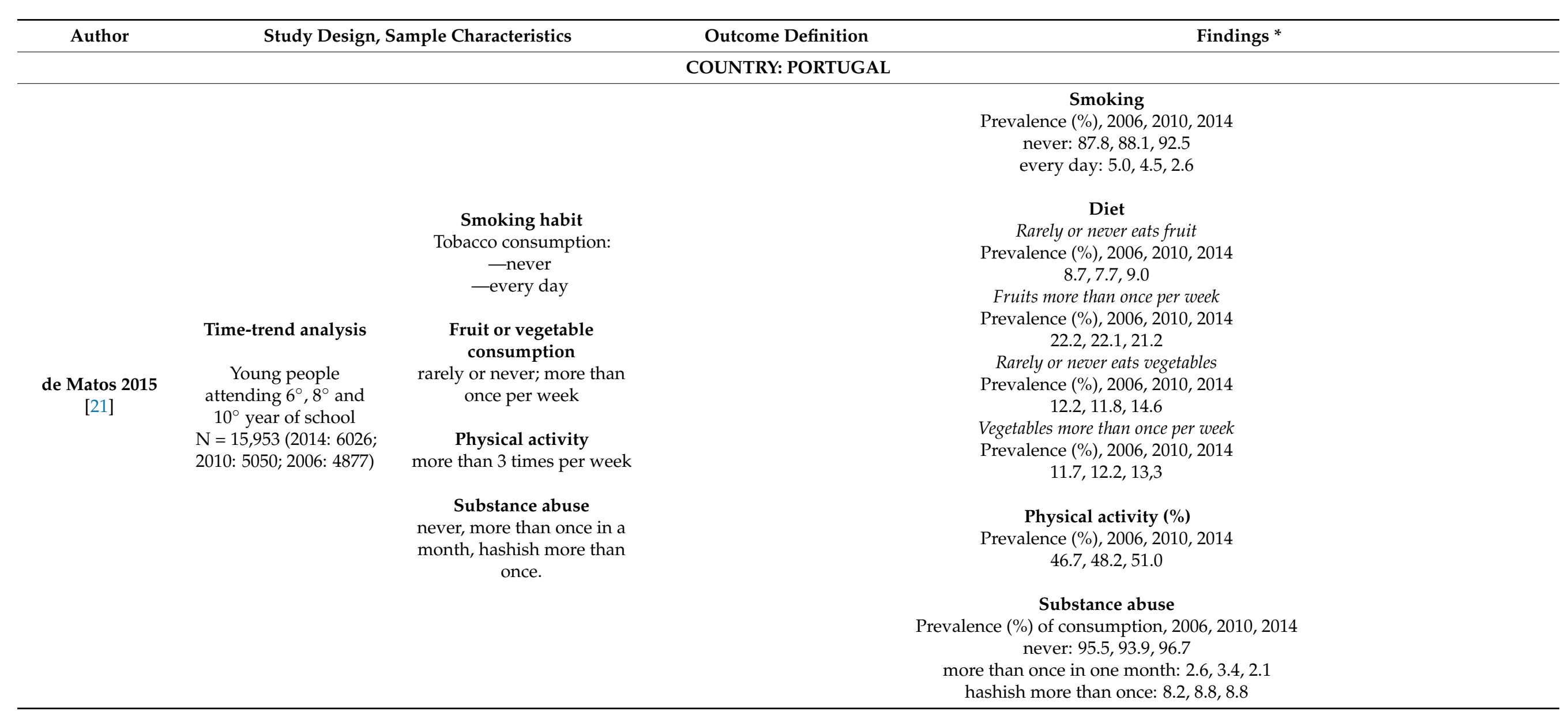


Table 1. Cont.

\begin{tabular}{|c|c|c|c|}
\hline Author & \multicolumn{2}{|c|}{ Study Design, Sample Characteristics } & Outcome Definition \\
\hline Silva $2020[38]$ & $\begin{array}{l}\text { Cross-sectional } \\
\text { World Mental Health } \\
\text { Survey Initiative } \\
\text { Portugal }(2008 / 09) \text { and } \\
\text { the National Mental } \\
\text { Health Survey } \\
\text { Follow-Up }(2015 / 16) \\
\text { Adults aged } 18+ \\
\quad \mathrm{N}=911\end{array}$ & $\begin{array}{l}\text { Drugs } \\
\text { use of psychotropic drugs in } \\
\text { the previous } 12 \text { months }\end{array}$ & $\begin{array}{c}\text { Drugs } \\
\text { Any psychotropic drug } \\
\text { Adjusted OR, 2015-16 vs. 2008-09 } \\
1.50 \text { (95\% CI:1.13-2.01) } \\
\text { Interaction age*year: } 18-49^{*} 2015-20161.95 \text { (95\% CI: 1.32-2.90) } \\
\text { Interaction gender*year: men*2015-2016 } 1.85 \text { (95\% CI: 1.08-3.17) } \\
\text { Antidepressant } \\
\text { Interaction age*year: } 18-49^{*} 2015-20161.68 \text { (95\% CI: 1.05-2.68) } \\
\text { Hypnotics/sedatives } \\
\text { 1.60 (95\% CI: } 1.14-2.25) \\
\left.\text { Interaction age*year: } 18-49^{*} 2015-20162.16 \text { (95\% CI: } 1.34-3.47\right) \\
\left.\text { Interaction gender }{ }^{*} \text { year: } \text { men }^{*} 2015-20162.60 \text { (95\% CI: } 1.36-4.98\right)\end{array}$ \\
\hline & & & COUNTRY: GREECE \\
\hline $\begin{array}{c}\text { Filippidis } 2014 \\
\text { [23] }\end{array}$ & $\begin{array}{c}\text { Cross-sectional } \\
\text { Hellas Health I, II and } \\
\text { IV } \\
\text { Adults aged 18+ } \\
\text { N = 3503 (2006: 1005; } \\
\text { 2008: 1490; 2011: } 1008)\end{array}$ & $\begin{array}{c}\text { Current smokers } \\
\text { those who smoke every day or } \\
\text { occasionally } \\
\text { Fruit and vegetable } \\
\text { consumption } \\
\text { daily number of portions } \\
\text { Physical activity } \\
\text { high, moderate, or low }\end{array}$ & $\begin{array}{c}\text { Smoking } \\
\text { Daily or occasional smokers } \\
\text { Difference 2011-2008-2006 (\%): }-11.56, p \text { for linear trend: } 0.014 \\
\text { By socioeconomic status } \\
\text { Higher: }-18.62, \mathrm{~ns} \\
\text { Middle: }-1.06, \mathrm{~ns} \\
\text { Lower: }-24.35, p=0.023 \\
\text { Diet } \\
\text { At least } 5 \text { daily portions of fruit and vegetables } \\
\text { Difference 2011-2008-2006 (\%): }-66.27, p \text { for linear trend: } 0.001 \\
\text { By socioeconomic status } \\
\text { Migher: }-68.79, p<0.001 \\
\text { Lower: }-81.76, p<0.001 \\
\text { Physical activity } \\
\text { High or moderate level of physical activity } \\
\text { Difference 2011-2008-2006 }(\%):+20.49 p \text { for linear trend: } 0.001 \\
\text { By socioeconomic status } \\
\text { Higher: }+10.66, \text { ns } \\
\text { Middle: }+22.44, p=0.001 \\
\text { Lower: }+23.02, p<0.001\end{array}$ \\
\hline
\end{tabular}


Table 1. Cont.

\begin{tabular}{|c|c|c|c|}
\hline Author & \multicolumn{2}{|c|}{ Study Design, Sample Characteristics } & Outcome Definition \\
\hline & & & COUNTRY: GREECE \\
\hline $\begin{array}{l}\text { Filippidis } 2017 \\
{[24]}\end{array}$ & $\begin{array}{c}\text { Cross-sectional } \\
\text { Hellas Health I, II, III, } \\
\text { IV, and V } \\
\text { Adults aged 18+ } \\
\text { N = 5504 (2006: 1005; } \\
\text { 2008: 1490; 2010: 1000; } \\
\text { 2011: 1008; 2015: 1001) }\end{array}$ & $\begin{array}{c}\text { Smoking habit } \\
\text { every day or occasionally } \\
\text { Fruit and vegetable } \\
\text { consumption } \\
\text { daily number of portions } \\
\text { Physical activity } \\
\text { high, moderate, or low }\end{array}$ & $\begin{array}{c}\text { Smoking } \\
\text { Current smokers } \\
\text { Prevalence (\%), } 2008 \text { and } 2015 \\
\text { 42.6 (95\% CI: } 40.0-45.1) 36.5 \text { (95\% CI: } 33.3-39.7) \\
\text { Diet } \\
\text { Adjusted risk ratio (RR) } 2015 \text { vs. } 2008: 0.86 \text { ( } 95 \% \text { CI: } 0.77-0.95) \\
\text { Low fruit/vegetable consumption } \\
\text { Prevalence (\%), } 2008 \text { and } 2015 \\
\text { 52.1 (95\% CI: } 49.6-54.7) 51.2 \text { (95\% CI: } 47.9-54.6) \\
\text { Adjusted risk ratio (RR) } 2015 \text { vs. } 2008: 1.00 \text { ( } 95 \% \text { CI: } 0.92-1.09) \\
\text { Physical activity } \\
\text { Sedentary lifestyle } \\
\text { Prevalence (\%), } 2006 \text { and } 2015 \\
\text { Adjusted risk ratio (RR), } 2015 \text { vs. } 2006: 0.69 \text { ( } 95 \% \text { CI: } 0.61-0.79)\end{array}$ \\
\hline \multirow[b]{2}{*}{$\begin{array}{c}\text { Madianos } 2014 \\
{[26]}\end{array}$} & Time-trend analysis & & \\
\hline & $\begin{array}{l}\text { Greek Population at } \\
\text { census } \\
\text { N =10,387,000 (1991), } \\
10,964,000(2001) \\
10,939,000(2011)\end{array}$ & $\begin{array}{l}\text { Alcohol per capita } \\
\text { consumption (liters) } \\
\text { Antidepressant consumption } \\
\text { (daily unit) }\end{array}$ & $\begin{array}{l}\text { Alcohol } \\
\text { Per capita consumption (liters), } 2005 \text { and 2011: } 9.24 \text { and } 8.80 \\
\text { Drugs } \\
\text { Daily unit consumption of antidepressants, } 2005 \text { and 2011: 215.40, 284.85 }\end{array}$ \\
\hline Sanidas 2018 [36] & $\begin{array}{l}\text { Retrospective study } \\
\text { Hospitalized patients } \\
\text { subjected to } \\
\text { cardiac catheterization } \\
\text { N = 3895 (2006-07: } \\
1228 ; \\
\text { 2011-15: } 2667)\end{array}$ & $\begin{array}{l}\text { Current smokers } \\
\text { at least } 1 \text { cigarette per day }\end{array}$ & $\begin{array}{c}\text { Smoking } \\
\text { Prevalence (\%) of smokers, 2006-2007 vs. 2011-2015: } 45.4 \text { vs. } 36.9, p=<0.001\end{array}$ \\
\hline
\end{tabular}


Table 1. Cont.

\begin{tabular}{|c|c|c|c|}
\hline Author & \multicolumn{2}{|c|}{ Study Design, Sample Characteristics } & Outcome Definition \\
\hline \multicolumn{4}{|r|}{ COUNTRY: GREECE } \\
\hline $\begin{array}{c}\text { Venetsanou } 2020 \\
{[41]}\end{array}$ & $\begin{array}{l}\text { Cross-sectional } \\
\text { Children attending } \\
\text { childcare centers (mean } \\
\text { age: } 52.72 \pm 3.55 \\
\text { months) } \\
\mathrm{N}=652(2009: 182 \\
\text { 2012: } 161 ; 2015: 165 ; \\
\text { 2018: } 144)\end{array}$ & $\begin{array}{c}\text { Physical activity } \\
\text { measured with Omron } \\
\text { Walking style pro HJ-720IT-E2 } \\
\text { pedometer }\end{array}$ & $\begin{array}{c}\text { Physical activity } \\
\text { Step counts, 2009, 2012, 2015, 2018 } \\
\text { Weekly: } 8032 \pm 2026,7816 \pm 2087,6708 \pm 2739,6943 \pm 2729 \\
\text { School-time: } 3646 \pm 1372,3459 \pm 1175,3233 \pm 1590,2991 \pm 1433 \\
\text { Leisure-time } 4906 \pm 1300,4899 \pm 1321,4026 \pm 1531,4312 \pm 1466 \\
\text { Weekend } 6700 \pm 2914,7112 \pm 2802,5676 \pm 3321,6031 \pm 3412 \\
\text { Statistically significant differences between cohort } \\
\text { School-time: } 2009 \text { vs. } 2018 \\
\text { Leisure-time: } 2009 \text { vs. } 2015,2009 \text { vs. } 2018,2012 \text { vs. } 2015 \\
\text { Weekend: } 2009 \text { vs. } 2015,2012 \text { vs. } 2015\end{array}$ \\
\hline \multicolumn{4}{|r|}{ Country: Multicenter } \\
\hline $\begin{array}{c}\text { Bosque-Prous } \\
2017 \text { [19] }\end{array}$ & $\begin{array}{l}\text { Cross-sectional } \\
\text { Economically active } \\
\text { adults: } 50-64 \text { years } \\
\mathrm{N}=25,479 \text { (2006: } 8016 \\
\text { 2013: } 17463)\end{array}$ & $\begin{array}{c}\text { Hazardous drinking } \\
\text { average daily consumption of } \\
>2 \text { and }>3 \text { alcoholic drinks in } \\
\text { the previous } 3 \text { months } \\
\text { Abstention } \\
\text { not drinking any alcoholic } \\
\text { beverage during the } 3 \text { months } \\
\text { prior to the interview }\end{array}$ & $\begin{array}{c}\text { Alcohol } \\
\text { Hazardous drinking } \\
\text { Changes in the prevalence (\%), 2006-2007 vs. } 2013 \\
\text { SPAIN } \\
\text { MEN: }-5.4 \text { (95\% CI: }-8.8--2.0) \\
\text { WOMEN: }-1.9 \text { (95\% CI: }-5.7--1.8) \\
\text { ITALY } \\
\text { MEN: }-5.6 \text { (95\% CI: }-9.1--2.2) \\
\text { WOMEN: }-1.4 \text { (95\% CI: }-4.7--1.9) \\
\\
\text { Adjusted prevalence ratio (PR), } 2013 \text { vs. } 2006-2007 \\
\text { SPAIN } \\
\text { MEN: PR }=0.42(95 \% \text { CI: } 0.23-0.81) \\
\text { WOMEN: PR }=0.67 \text { ( } 95 \% \text { CI: } 0.24-1.97) \\
\text { ITALY } \\
\text { MEN: PR }=0.44 \text { (95\% CI: } 0.27-0.79) \\
\text { WOMEN: PR }=0.63 \text { ( } 95 \% \text { CI: } 0.21-1.70)\end{array}$ \\
\hline
\end{tabular}


Table 1. Cont.

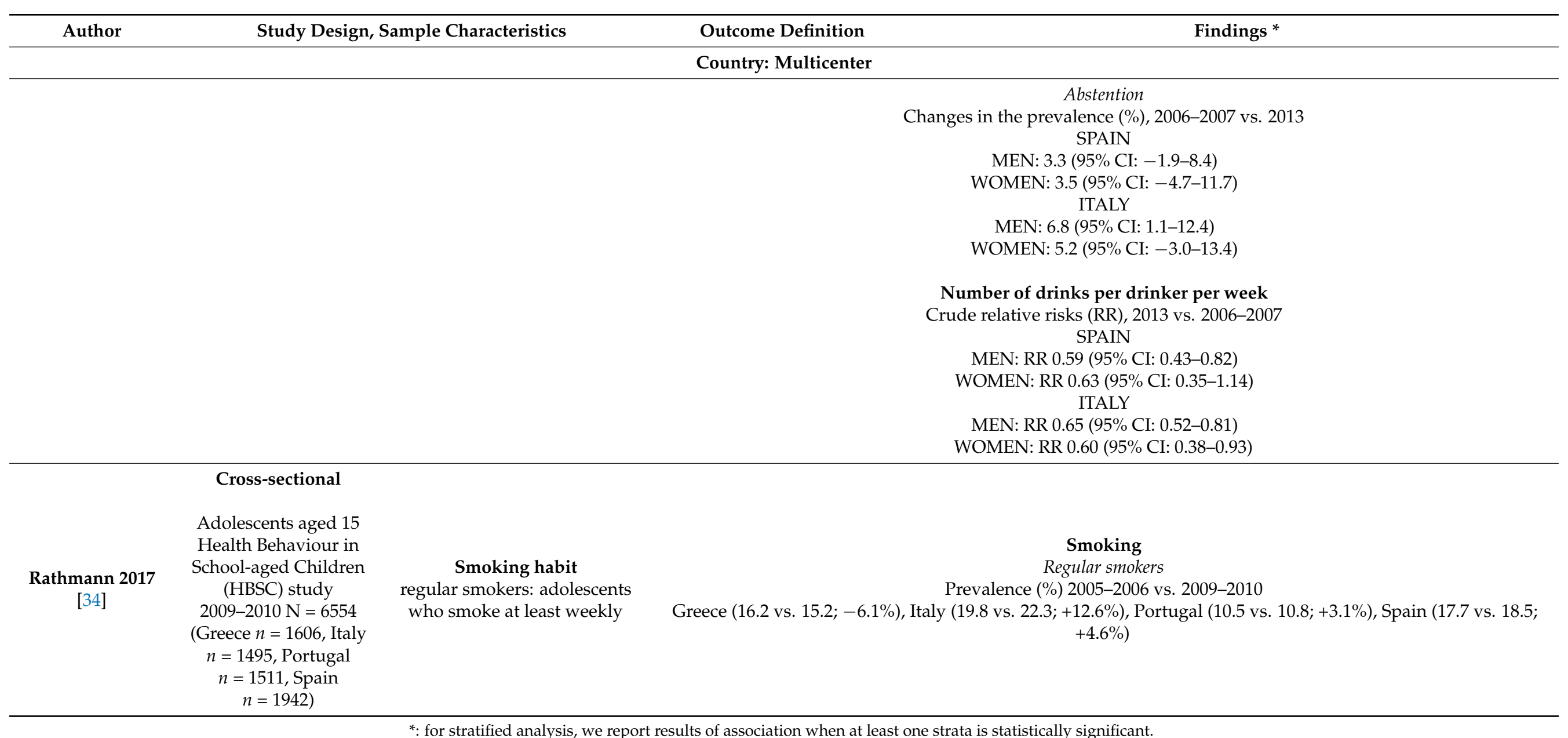

*: for stratified analysis, we report results of association when at least one strata is statistically significant. 
Table 2. Variation of behavior influencing health status during or after the 2008 financial crisis.

\begin{tabular}{|c|c|c|c|c|c|c|c|}
\hline First Author & Country & Alcohol & Smoking & $\begin{array}{l}\text { Healthy } \\
\text { Diet }\end{array}$ & $\begin{array}{l}\text { Physical } \\
\text { Activity }\end{array}$ & Drugs & $\begin{array}{c}\text { Substance } \\
\text { Abuse }\end{array}$ \\
\hline Aguilar-Palacio 2015 [13] & Spain & - & - & & & & \\
\hline Arroyo 2018 [15] & Spain & & & & & - & \\
\hline Bartoll 2015 [16] & Spain & - & + & - & + & - & \\
\hline Blázquez-Fernández 2019 [17] & Spain & $=$ & & & & & \\
\hline Bosque-Prous 2017 [19] & Spain & - & & & & & \\
\hline Colell 2015 [20] & Spain & - & & & & + & - \\
\hline Diaz-Mendez 2019 [22] & Spain & & & - & & & \\
\hline Garcia Mayor 2020 [25] & Spain & - & - & - & + & & \\
\hline Marquez-Calderon 2014 [27] & Spain & - & - & & - & + & - \\
\hline Martin Bassols 2016 [28] & Spain & - & $=$ & & & & - \\
\hline Moreno Lostao 2019 [30] & Spain & & $\mathrm{N} / \mathrm{A}$ & & $\mathrm{N} / \mathrm{A}$ & & \\
\hline Perez-Romero 2016 [31] & Spain & & & & & + & \\
\hline Rajmil 2013 [33] & Spain & & & - & - & & \\
\hline Rathmann 2017 [34] & Spain & & + & & & & \\
\hline Regidor 2019 [35] & Spain & - & - & + & + & & \\
\hline Spijker 2018 [39] & Spain & & & & - & & \\
\hline Trujillo-Aleman 2019 [40] & Spain & & - & & & & \\
\hline Zapata Moya 2020 [42] & Spain & & & & & + & \\
\hline Zozaya 2020 [43] & Spain & - & - & & & & \\
\hline Bonaccio 2014 [18] & Italy & + & & - & & & \\
\hline Bosque-Prous 2017 [19] & Italy & - & & & & & \\
\hline Mattei 2017 [29] & Italy & - & + & & & & \\
\hline Petrelli 2017 [32] & Italy & & - & & & & \\
\hline Rathmann 2017 [34] & Italy & & + & & & & \\
\hline Sarti 2018 [37] & Italy & - & - & $=$ & - & & \\
\hline Alves 2019 [14] & Portugal & & & - & & & \\
\hline de Matos 2015 [21] & Portugal & & - & - & + & & - \\
\hline Rathmann 2017 [34] & Portugal & & + & & & & \\
\hline Silva 2020 [38] & Portugal & & & & & + & \\
\hline Filippidis 2014 [23] & Greece & & - & - & + & & \\
\hline Filippidis 2017 [24] & Greece & & - & $=$ & + & & \\
\hline Madianos 2014 [26] & Greece & - & & & & + & \\
\hline Sanidas 2018 [36] & Greece & & - & & & & \\
\hline Venetsanou 2020 [41] & Greece & & & & - & & \\
\hline Rathmann 2017 [34] & Greece & & - & & & & \\
\hline
\end{tabular}

Note: statistically significant decrease (-); increase (+) or no variation (=) in the prevalence of healthy behavior; N/A: not applicable.

\subsubsection{The Financial Crisis and Smoking}

Similarly to alcohol consumption, smoking habit also appeared to decrease markedly in most studies conducted in different settings or subgroups: young Spanish men aged 16-24 [13], adolescents in Portugal [21] and Spain [43], and adults in Greece [23,24] and Spain (only men) [25]. A reduction in smoking prevalence was observed in Spain also when stratifying by the size of the municipality [30], in a sample of patients hospitalized for cardiac catheterization [36], and in a sample of Spanish mothers [40]. A few studies 
reported a slight decrease [27,32], no difference [37], or a slight increase in the percentage of smokers after or during the crisis, for only women [16] in Spain and for both sexes in Italy [29,34], Portugal, and Spain [34].

Regarding socioeconomic status, controversial associations were reported during or after the crisis: a reduction in inequalities was identified by some studies, where percentages of daily or occasional smokers decreased more for those with a lower educational level [16], lower socioeconomic status [23], or having a manual occupation [40]. Conversely, other studies found an increase in socioeconomic inequalities due to an increase in smoking consumption among the unemployed $[13,28,37,43]$ and among people belonging to a lower social class [25].

\subsubsection{The Financial Crisis and Healthy Diet}

Generally speaking, a deterioration in the quality of diet was observed during and after the crisis. Several studies showed a reduction in the consumption of meat [16], fish [14,22], fruits $[14,16,21-25]$, and vegetables [14,22-24] in Spain and Portugal, although in Portugal the consumption of vegetables increased among young people [21], and legumes were more frequently consumed $[14,16]$. Sweets and desserts were more often consumed by adults in Portugal [14] and Spain [16].

Several studies reported an increase in socioeconomic inequalities in healthy diet, especially regarding fruits and vegetables. In Spain, the probability of declaring eating fruit daily decreased more among unemployed men and least educated men, and the probability of declaring eating vegetables daily among unemployed men and women and the least educated women [16]. Two other Spanish studies reported an increase in inequalities in the consumption of fruits and vegetables [22,25].

Similar results were observed regarding fish consumption [22]. During the crisis in Italy, the socioeconomic differences in adherence to the Mediterranean diet widened, becoming less probable among people with a low wealth index score, those with a lower education level, and those performing manual labor [18]. One study conducted in Spain on young people below the age of 15 reported that the prevalence of junk food consumption increased in families with low maternal education level [33].

\subsubsection{The Financial Crisis and Physical Activity}

Most of the studies highlighted an increase in physical activity in the adult population when comparing the periods after vs before the financial crisis, which was more robust and statistically significant in Spain $[25,35]$ and Greece $[23,24]$, and slighter in Portugal [21], while a limited reduction was observed in the few studies in adults [37] and in children $[33,41]$.

Two Spanish studies reported an increase in socioeconomic inequalities in physical activity during and after the crisis. In one, an increase in the prevalence of physical activity was observed in all social classes, but this was slighter in the lower class, resulting in an increase in socioeconomic differences [25]. The other study observed that physical activity increased during or after the crisis among more educated women and decreased among the less educated, causing a widening of socioeconomic inequalities [16].

Instead, a Greek study showed a reduction in inequalities due to a significant increase in percentages of adults only, with those with a middle or lower socioeconomic status reporting high or moderate level of physical activity [24].

\subsubsection{The Financial Crisis and Use of Antidepressant, Anxiolytic, and/or} Antipsychotic Drugs

Most studies reported an increase in any type of psychotropic drug use among all individuals considered [31,42] or only among women [16]. In a Spanish study performed on the economically active population, heavy use of hypnotics/sedatives among men and women increased in the period examined [20]. In a population study conducted in Portugal, the odds of consuming any psychotropic drug was estimated to be 1.5 times higher than before the crisis, and when evaluating the interaction effect of the year with sex and 
age, men and younger individuals reported higher odds of consuming any psychotropic drug [38]. Bartoll et al. [16] observed a stable trend in tranquilizer tablet use among men and a decrease among women.

This general increasing trend was not homogeneously reported for all psychotropic drugs: specifically, Marquez Calderon et al. [27] found an increase in sedative, tranquilizer, and hypnotic drugs, while antidepressant use decreased. According to Arroyo [15], only sedative use increased, and Madianos et al. evaluated only antidepressant use, finding an increase in its use in Greece [26].

When considering socioeconomic status, according to Arroyo et al. [15], the probability of consuming antidepressants or sedatives depended on employment status: in the case of individuals in short-term unemployment, both men and women showed that between 2006-2007 and 2011-2012, there was an increase in the risk of using sedatives. However, this increase was greater for women than for men. For the long-term unemployed, however, the differences between both sexes widened between 2006-2007 and 2011-2012: the risk of using sedatives in women increased, whereas it decreased in men, while antidepressant consumption decreased overall, and more markedly among short- and long-term unemployed subjects. Regarding education level, there was an increase in the intake of tranquilizers among those without any qualification and a slight decrease for men with high secondary education; among women, there was a drop-in intake, which was greater among those employed and those without any qualification [16].

Finally, an increase in psychotropic drug consumption was observed among households whose socioeconomic status was most affected by the crisis [42].

\subsubsection{The Financial Crisis and Substance Abuse}

One of the included studies described a stable or a slight downward trend in drug use [28]. A study performed in Portugal on adolescents reported a slight decrease in monthly drug use [21]. A decrease in cocaine, marijuana, ecstasy, and hard drug use caused by the economic downturn, which could have affected the prices of these drugs, was observed by a Spanish study [28]. According to another Spanish study, overall cannabis use remained stable during the crisis, but unemployed men and women were more likely to have increased sporadic use compared to their employed counterparts [20]. Given a 10\% increase in the provincial unemployment rate in Spain, an increase in the probability of using marijuana and cocaine in the last 30 days and also over the previous 12 months was observed [28].

\section{Discussion}

Since the financial crisis of 2008, southern European countries, in particular Italy, Greece, Spain, and Portugal, have been affected by the economic recession [44-46].

The research articles included in our review, published between 2011 and 2020, concerned the impact of the crisis on unhealthy behaviors, such as smoking habit $[13,16,21,23-$ 25,27-30,32], alcohol consumption [13,16-20,25-29,35,37,43], antidepressant/anxiolytic/ antipsychotic use $[15,16,20,26,27,31,38,42]$, and substance abuse $[20,21,27,28]$ and on healthy behaviors, such as physical activity $[16,21,23-25,27,30,33,35]$. The studies on diet examined the impact of the crisis both on favorable and unfavorable eating behavior, a classification depending on the type of food considered for analyses [14,16,18,21-25,33,35,37].

According to the findings of our extensive review, we observed controversial effects on healthy behaviors in the period characterized by the 2008 financial crisis.

In general, the studies found that alcohol consumption $[13,16,19,20,25-29,35,43]$ and substance abuse $[21,28]$ decreased during or after the Great Recession, while psychotropic drug use increased $[16,20,31,38,42]$.

A deterioration in proper eating habits was also observed [14,16,21-25], but some comments regarding diet are necessary. In fact, although most of the studies report an overall worsening of eating behavior during the crisis, the evaluation of its impact is more complex than it is for the other lifestyle-related behaviors. A reduction in the consumption 
of fish and meat, probably due to the reduction in available income, was observed, as was a reduction in fruit and vegetable consumption; both of these phenomena could cause a shift toward a worse diet. The crisis effect could have also contributed to determine the decrease in consumption of fruits and vegetables and the increase in junk food, sweets, and dessert consumption. Social inequality in proper eating habits generally increased because of the economic crisis $[16,18,22,25,33]$ : several studies observed a decrease in the consumption of fruits and vegetables and an increase in junk food consumption among people with a low socioeconomic status.

However, even before the crisis, a decline in adherence to the Mediterranean diet had been observed in the younger population, so that a reduction in the mean consumption of fruit and vegetables was expected. Therefore, this decline may not be solely attributed to the economic crisis [23].

Most studies also showed an overall decrease in alcohol [13,16,19,20,25-28,35,37] and tobacco $[13,21,23-25,27,32,34-37,40,43]$ consumption, while some highlighted an increase in tobacco consumption $[16,29,34]$. However, the pre- to post crisis variation in drinking and smoking habit were heterogeneous across socioeconomic levels, depending on the contexts and the dimension used to define socioeconomic status. For example, a study showed an increase in heavy alcohol consumption during the crisis among individuals with the lowest education level [16].

Less income available to purchase alcohol might have been behind the decrease in heavy drinking, while binge drinking could have increased as a means to deal with anxiety and emotional distress related to job loss, whether real or threatened, and to financial hardship [20]. However, it has been argued that a process of change in the pattern of alcohol use is taking place in Mediterranean countries such as Spain, where alcohol has traditionally been embedded in daily life, with wine drunk regularly with meals [20].

The decrease in tobacco use during times of economic downturn is related to price increases; it is likely that tobacco control measures may have interacted synergistically with the decline in disposable income. Therefore, austerity may have been a driving force in the decline among low-income individuals, along with the other public health measures [23].

Most of the studies found a slight increase in physical activity during the crisis $[16,21,23-$ $25,35]$, while other studies found a slight increase in sedentary habits $[27,33,37,39,41]$. There was an increase in socioeconomic differences in the prevalence of physical activity [16,25], with a heterogeneous gradient according to socioeconomic status.

The reduction in substance abuse observed during the crisis was slight, and generally referred to soft drugs $[20,21,27,28]$, without any significant differences in terms of socioeconomic status.

Most studies reported an increase in the use of any psychotropic drug [20,26,27,31,38,42].

The decreased availability of income during the recession may have determined a reduction in the purchase of tobacco, alcohol, and drugs, but also of more expensive and healthier food. On the contrary, the increase in the use of psychotropic drugs can be considered a kind of coping mechanism against the insecurity and stress related to the economic crisis.

Our review appears to confirm previous evidence $[47,48]$ that the financial crisis had an impact on socioeconomic inequalities and that negative effects on health tended to be more pronounced among the culturally, economically, and socially disadvantaged.

The low socioeconomic strata experienced inequalities in access to cultural and material resources (e.g., education, working conditions, income), which determined worse health and limited access to appropriate health care. These inequalities grew over the course of the global crisis, and the recession period could have accelerated the accumulation of such disadvantages $[10,49,50]$.

The crisis itself may have played an independent, additional role, acting as a chronic stressor. Furthermore, it is possible that unemployed people and those at risk of unemployment or who experienced work instability may have had less time to dedicate to themselves and to their lifestyle, including food choice and physical activity [29]. 
In this scenario, the COVID-19 pandemic has exacerbated inequalities with a cumulative effect of the risks [51]. In England, as already shown in the Marmot Review, COVID-19 infection and mortality risks have been much higher for those living in more deprived areas, in overcrowded housing, in key workers in close proximity to others, in those from minority groups, in those with underlying health conditions, and in those who are older and/or male [52]. Furthermore, due to the effects of the mobility restrictions and the periods of lockdown as well as to the impoverishment of many sections of the population, it is reasonable to expect a further deterioration in lifestyles, especially among the people most affected by the economic crisis generated by the pandemic [53].

In fact, most countries were forced to introduce confinement measures to minimize the propagation of the SARS-CoV-2 virus, and for many people, it was difficult to maintain a healthy lifestyle, in particular a proper diet, regular physical exercise, quality of sleep, and limited smoking and alcohol consumption [53-69]. However, similar to the analysis reporting on the 2008 recession, the effects on diet are not unidirectional. In fact, many people used the period of home isolation to improve their eating habits and to limit dietary excesses and bad eating behaviors. Having the opportunity to devote time daily to having breakfast and to cooking meals resulted in an increase in the consumption of fruit, vegetables, and pulses $[56,60,64,67,68]$, and a general decrease in alcohol consumption $[56,66,67]$.

\section{Strengths and Limitations}

Our review of the impact of the 2008 financial crisis on many health behaviors aimed to shed light on the links between changes in habits and health outcomes, an issue that has not yet been systematically investigated [3]. The focus on four southern European countries represents an added value, as they were hit harder by the crisis than were other European countries.

By focusing exclusively on health outcomes, our study did not look at the impact of the crisis on health systems, such as shortages in the health workforce or in medical supplies, for which several studies have shown a negative trend during the financial crisis [3,70,71].

The exact moment the financial crisis began is difficult to establish, and some studies, reporting data on different countries, have defined the duration of the crisis differently; this may have had an impact on the homogeneity of the reported results. The included studies had a high risk of bias in exposure and outcome assessment due to the study design, use of self-reported measures, and the lack of adjusting for potential confounding factors. Further, some observational studies did not apply any statistical tests $[21,22,26,27,32,34,37,39,43]$. Most importantly, although the studies included in our review investigated changes in population health status and health behaviors associated with the Great Recession, it cannot be established whether this was a causal relationship.

Due to the nature of the data of most of the included studies, which were not designed to measure exposure at the individual level, no causal relationship between the economic recession and changes in lifestyles can be established. We can, however, state that these changes occurred after the crisis. In fact, although a causal association between the financial crisis and trends in risk factors seems reasonable, we cannot exclude unmeasured confounding, which would provide alternative explanations for the observed trends. On the other hand, the financial crisis is a natural experiment at the population level and the possibility of its effects on the findings may be supposed [23].

Finally, self-reported information collected by questionnaire, as was the case for most of the included studies, may have been affected by information bias.

\section{Conclusions}

Our results seem to show that the crisis has had a negative effect on eating habits and a positive effect on alcohol consumption and on smoking, the consequence of mechanisms probably determined by decreased available income. Psychophysical stress linked to unemployment and job loss, as well as the worsening mental health observed in nu- 
merous studies, could explain the increase in the consumption of antidepressant and anxiolytic drugs.

These results suggest the need to implement health policies aimed at monitoring risk behaviors and for interventions aimed at contrasting the effects of the financial crisis in the countries studied. Inequalities in health behaviors should also be a priority area for action. Financial recommitment to public health system should accompany a substantial commitment to tackling the social determinants of poor health and wellbeing.

The identification of lifestyles and socioeconomic inequalities produced by the 2008 crisis may facilitate the understanding and the response to the possible effects of the current COVID-19 crisis.

The current pandemic provides harsh lessons on the societal vulnerabilities that arise from inequality. Investing in young people and supporting long-deprived regions and sectors of society are arguably the most powerful ways to break the chain of inequality transmitted from generation to generation. Adopting a broadened, equity-focused approach to population health should be an essential part of building a more resilient society that is better prepared to weather future pandemics.

Supplementary Materials: The following are available online at https:/ /www.mdpi.com/article/10 .3390/ijerph18168734/s1, Table S1: MEDLINE Search—Updated November 2020, Table S2: Risk of bias assessment for all the selected studies.

Author Contributions: Conceptualization, A.P. and A.D.N.; Methodology, S.V. and M.S.S.; Software, M.S.S. and R.A.; Formal Analysis, M.S.S., A.D.N., R.A., S.V. and A.P.; Data Curation, M.S.S., A.D.N., R.A., S.V. and A.P.; Writing-Original Draft Preparation, M.S.S., A.D.N., R.A., S.V. and A.P.; WritingReview and Editing, A.D.N., S.V., C.M. and A.P.; Project Administration, A.P. All authors have read and agreed to the published version of the manuscript.

Funding: This research received no external funding.

Institutional Review Board Statement: Not applicable.

Informed Consent Statement: Not applicable.

Acknowledgments: We gratefully acknowledge Jacqueline M. Costa for the contribution in translation and English language editing.

Conflicts of Interest: The authors declare no conflict of interest.

\section{References}

1. International Monetary Fund. World Economic Outlook; International Monetary Fund: Washington, DC, USA, 2011.

2. Karanikolos, M.; Mladovsky, P.; Cylus, J.; Thomson, S.; Basu, S.; Stuckler, D.; Mackenbach, J.P.; McKee, M. Financial crisis, austerity, and health in Europe. Lancet 2013, 381, 1323-1331. [CrossRef]

3. Parmar, D.; Stavropoulou, C.; Ioannidis, J.P. Health outcomes during the 2008 financial crisis in Europe: Systematic literature review. BMJ 2016, 354, i4588. [CrossRef] [PubMed]

4. Karanikolos, M.; Heino, P.; McKee, M.; Stuckler, D.; Legido-Quigley, H. Effects of the global financial crisis on health in high-income Oecd countries: A Narrative Review. Int. J. Health Serv. 2016, 46, 208-240. [CrossRef] [PubMed]

5. WHO. Impact of the Economic Crises on Mental Health; World Health Organisation: Copenhagen, Denmark, 2011; pp. 1-34.

6. Zavras, D.; Zavras, A.I.; Kyriopoulos, I.I.; Kyriopoulos, J. Economic crisis, austerity and unmet healthcare needs: The case of Greece. BMC Health Serv. Res. 2016, 16, 309. [CrossRef] [PubMed]

7. Petrelli, A.; Rosano, A.; Rossi, A.; Mirisola, C.; Cislaghi, C. The geography and economics of forgoing medical examinations or therapeutic treatments in Italy during the economic crisis. BMC Public Health 2019, 19, 1202. [CrossRef] [PubMed]

8. Mackenbach, J.P.; Kulhánová, I.; Artnik, B.; Bopp, M.; Borrell, C.; Clemens, T.; Costa, G.; Dibben, C.; Kalediene, R.; Lundberg, O.; et al. Changes in mortality inequalities over two decades: Register based study of European countries. BMJ 2016, 353, i732. [CrossRef]

9. Heggebø, K.; Tøge, A.G.; Dahl, E.; Berg, J.E. Socioeconomic inequalities in health during the Great Recession: A scoping review of the research literature. Scand. J. Public Health 2019, 47, 635-654. [CrossRef]

10. de Goeij, M.C.; Suhrcke, M.; Toffolutti, V.; van de Mheen, D.; Schoenmakers, T.M.; Kunst, A.E. How economic crises affect alcohol consumption and alcohol-related health problems: A realist systematic review. Soc. Sci. Med. 2015, 131, 131-146. [CrossRef]

11. Nagelhout, G.E.; Hummel, K.; de Goeij, M.C.M.; de Vries, H.; Kaner, E.; Lemmens, P. How economic recessions and unemployment affect illegal drug use: A systematic realist literature review. Int. J. Drug Policy 2017, 44, 69-83. [CrossRef] 
12. Wells, G.; Shea, B.; O'Connell, D.; Peterson, J.; Welch, V.; Losos, M.; Tugwell, P. The Newcastle-Ottawa Scale (NOS) for Assessing the Quality of Non-Randomized Studies in Meta-Analysis. 2000. Available online: http://www.ohri.ca/programs/clinical_ epidemiology / oxford.asp (accessed on 12 August 2021).

13. Aguilar-Palacio, I.; Carrera-Lasfuentes, P.; Rabanaque, M.J. Youth unemployment and economic recession in Spain: Influence on health and lifestyles in young people (16-24 years old). Int. J. Public Health 2015, 60, 427-435. [CrossRef]

14. Alves, R.; Perelman, J. Dietary changes during the Great Recession in Portugal: Comparing the 2005/2006 and the 2014 health surveys. Public Health Nutr. 2019, 22, 1971-1978. [CrossRef]

15. Arroyo, E.; Cabrera-Leon, A.; Renart, G.; Saurina, C.; Serra Saurina, L.; Daponte, A.; Saez, M. Did psychotropic drug consumption increase during the 2008 financial crisis? A cross-sectional population-based study in Spain. BMJ Open 2019, 9, e021440. [CrossRef] [PubMed]

16. Bartoll, X.; Toffolutti, V.; Malmusi, D.; Palencia, L.; Borrell, C.; Suhrcke, M. Health and health behaviours before and during the Great Recession, overall and by socioeconomic status, using data from four repeated cross-sectional health surveys in Spain (2001-2012). BMC Public Health 2015, 15, 865. [CrossRef] [PubMed]

17. Blázquez-Fernández, C.; Cantarero-Prieto, D.; Perez, P. Do the unemployed hit the bottle during economic downturns? An empirical approach for Spain. BMC Public Health 2019, 19, 523. [CrossRef] [PubMed]

18. Bonaccio, M.; Di Castelnuovo, A.; Bonanni, A.; Costanzo, S.; De Lucia, F.; Persichillo, M.; Zito, F.; Donati, M.B.; de Gaetano, G.; Iacoviello, L. Decline of the Mediterranean diet at a time of economic crisis. Results from the Moli-sani study. Nutr. Metab. Cardiovasc. Dis. 2014, 24, 853-860. [CrossRef]

19. Bosque-Prous, M.; Kunst, A.E.; Brugal, M.T.; Espelt, A. Changes in alcohol consumption in the 50- to 64-year-old European economically active population during an economic crisis. Eur. J. Public Health 2017, 27, 711-716. [CrossRef]

20. Colell, E.; Sanchez-Niubo, A.; Delclos, G.L.; Benavides, F.G.; Domingo-Salvany, A. Economic crisis and changes in drug use in the Spanish economically active population. Addiction 2015, 110, 1129-1137. [CrossRef]

21. de Matos, M.G.; Reis, M.; Camacho, I.; Simoes, C.; Gomez-Baya, D.; Mota, C.; Tome, G.; Gaspar, T.; do Ceu Machado, M. In times of economic recession, did Portuguese adolescents stay healthy so far, but no longer happy? Arq. Med. 2015, 29, 116-122.

22. Diaz-Mendez, C.; Garcia-Espejo, I. Social Inequalities in Following Official Guidelines on Healthy Diet During the Period of Economic Crisis in Spain. Int. J. Health Serv. 2019, 49, 582-605. [CrossRef]

23. Filippidis, F.T.; Schoretsaniti, S.; Dimitrakaki, C.; Vardavas, C.I.; Behrakis, P.; Connolly, G.N.; Tountas, Y. Trends in cardiovascular risk factors in Greece before and during the financial crisis: The impact of social disparities. Eur. J. Public Health 2014, 24, 974-979. [CrossRef]

24. Filippidis, F.T.; Gerovasili, V.; Millett, C.; Tountas, Y. Medium-term impact of the economic crisis on mortality, health-related behaviours and access to healthcare in Greece. Sci. Rep. 2017, 7, 46423. [CrossRef]

25. Garcia-Mayor, J.; Moreno-Llamas, A.; De la Cruz-Sanchez, E. Inequalities in the long-term impact of the economic recession on preventive healthcare use and health-related lifestyle in Spain (2006-2017). Health Soc. Care Community 2021, 29, 42-55. [CrossRef]

26. Madianos, M.G.; Alexiou, T.; Patelakis, A.; Economou, M. Suicide, unemployment and other socioeconomic factors: Evidence from the economic crisis in Greece. Eur. J. Psychiat. 2014, 28, 39-49. [CrossRef]

27. Marquez-Calderon, S.; Villegas-Portero, R.; Gosalbes Soler, V.; Martinez-Pecino, F. Health promotion and prevention in the economic crisis: The role of the health sector. SESPAS report 2014. Gac. Sanit. 2014, 28, 116-123. [PubMed]

28. Martin Bassols, N.; Vall Castello, J. Effects of the great recession on drugs consumption in Spain. Econ. Hum. Biol. 2016, 22, 103-116. [CrossRef]

29. Mattei, G.; De Vogli, R.; Ferrari, S.; Pingani, L.; Rigatelli, M.; Galeazzi, G.M. Impact of the economic crisis on health-related behaviors in Italy. Int. J. Soc. Psychiatry 2017, 63, 649-656. [CrossRef] [PubMed]

30. Moreno-Lostao, A.; Guerras, J.M.; Lostao, L.; de la Fuente, L.; Martinez, D.; Rodriguez-Artalejo, F.; Regidor, E. Cardiovascular mortality and risk behaviours by degree of urbanization before, during and after the economic crisis in Spain. BMC Public Health 2019, 19, 1109. [CrossRef] [PubMed]

31. Perez-Romero, S.; Gascon-Canovas, J.J.; de la Cruz-Sanchez, E.; Sanchez-Ruiz, J.F.; Parra-Hidalgo, P.; Monteagudo-Piqueras, O. Economic recession (2006-2012) and changes in the health status of the Spanish population. Salud Publica Mex 2016, 58, 41-48. [PubMed]

32. Petrelli, A.; Di Napoli, A.; Rossi, A.; Costanzo, G.; Mirisola, C.; Gargiulo, L. The variation in the health status of immigrants and Italians during the global crisis and the role of socioeconomic factors. Int. J. Equity Health 2017, 16, 98. [CrossRef] [PubMed]

33. Rajmil, L.; Medina-Bustos, A.; Fernandez De Sanmamed, M.J.; Mompart-Penina, A. Impact of the economic crisis on children's health in Catalonia: A before-after approach. BMJ Open 2013, 3, e003286. [CrossRef]

34. Rathmann, K.; Pfortner, T.K.; Elgar, F.J.; Hurrelmann, K.; Richter, M. The great recession, adolescent smoking, and smoking inequalities: What role does youth unemployment play in 24 European Countries? Nicotine Tob. Res. 2017, 19, 1284-1291. [CrossRef] [PubMed]

35. Regidor, E.; Albaladejo, R.; Mateo, A.; de la Fuente, L.; Barrio, G.; Ortega, P. Macroeconomic fluctuations, changes in lifestyles and mortality from diabetes: A quasi experimental study. J. Epidemiol. Community Health 2019, 73, 317-323. [CrossRef] [PubMed]

36. Sanidas, E.A.; Papaioannou, T.G.; Papadopoulos, D.P.; Tatsi, K.; Velliou, M.; Dalianis, N.; Paizis, I.; Anastasiadis, G.; Kelepesis, G.; Bonou, M.; et al. The impact of financial crisis on coronary artery disease burden in Greece. Hellenic J. Cardiol. 2019, 60, 185-188. [CrossRef] [PubMed] 
37. Sarti, S.; Terraneo, M.; Tognetti Bordogna, M. Stili di vita nell'Italia della crisi: Il cambiamento nelle abitudini insalubri secondo le condizioni lavorative. Sociologia del Lavoro 2018, 150, 23-48. [CrossRef]

38. Silva, M.; Antunes, A.; Azeredo-Lopes, S.; Cardoso, G.; Xavier, M.; Saraceno, B.; Caldas-De-Almeida, J.M. How did the use of psychotropic drugs change during the Great Recession in Portugal? A follow-up to the National Mental Health Survey. BMC Psychiatry 2020, 20, 215. [CrossRef] [PubMed]

39. Spijker, J.; Zueras, P. Socioeconomic health inequalities in the Catalan population aged 50+ during the last economic crisis. Rev. Esp. Salud Publica 2018, 28, 92.

40. Trujillo-Aleman, S.; Perez, G.; Puig-Barrachina, V.; Gotsens, M.; Reynolds, J.; Rueda, S.; Borrell, C. Inequalities in health and health behaviours between couple and lone mothers before and during the financial crisis in Spain (2003-2012). SSM Popul. Health 2019, 7, 100367. [CrossRef]

41. Venetsanou, F.; Emmanouilidou, K.; Kouli, O.; Bebetsos, E.; Comoutos, N.; Kambas, A. Physical activity and sedentary behaviors of young children: Trends from 2009 to 2018. Int. J. Environ. Res. Public Health 2020, 17, 1645. [CrossRef]

42. Zapata Moya, Á.R.; Navarro Yáñez, C.J. Urban regeneration policies and mental health in a context of economic crisis in Andalusia (Spain). J. House Built. Environ. 2021, 36, 393-405. [CrossRef]

43. Zozaya. N.; Vallejo, L. The effect of the economic crisis on adolescents' perceived health and risk behaviors: A multilevel analysis. Int. J. Environ. Res. Public Health 2020, 17, 643. [CrossRef]

44. Stuckler, D.; Basu, S.; Suhrcke, M.; Coutts, A.; McKee, M. The public health effect of economic crises and alternative policy responses in Europe:an empirical analysis. Lancet 2009, 374, 315-323. [CrossRef]

45. Quaglio, G.; Karapiperis, T.; Van Woensel, L.; Arnold, E.; McDaid, D. Austerity and health in Europe. Health Policy 2013, 113, 13-19. [CrossRef]

46. Kentikelenis, A.; Karanikolos, M.; Papanicolas, I.; Basu, S.; McKee, M.; Stuckler, D. Health effects of financial crisis: Omens of a Greek tragedy. Lancet 2011, 378, 1457-1458. [CrossRef]

47. Kondo, N.; Subramanian, S.V.; Kawachi, I.; Takeda, Y.; Yamagata, Z. Economic recession and health inequalities in Japan: Analysis with a national sample, 1986-2001. J. Epidemiol. Community Health 2008, 62, 869-875. [CrossRef] [PubMed]

48. Marmot, M.G.; Allen, J.; Bell, R.; Bloomer, E.; Goldblatt, P. WHO European review of social determinants of health and the health divide. Lancet 2012, 380, 1011-1029. [CrossRef]

49. Merton, R.K. The Matthew Effect in Science: The renard and communication systems of science are considered. Science 1968, 159, 56-63. [CrossRef] [PubMed]

50. Di Prete, T.A.; Eirich, G.M. Cumulative advantage as a mechanism for inequality: A review of theoretical and empirical developments. Ann. Rev. Sociol. 2006, 32, 271-297. [CrossRef]

51. The Lancet Public Health. COVID-19-break the cycle of inequality. Lancet Public Health 2021, 6, e82. [CrossRef]

52. Marmot, M.; Allen, J.; Goldblatt, P.; Herd, E.; Morrison, J. Build Back Fairer: The COVID-19 Marmot Review. The Pandemic, Socioeconomic and Health Inequalities in England; Institute of Health Equity: London, UK, 2020.

53. ISS-Epicentro. COVID-19: Healthy Lifestyles also during the Emergency. Available online: https://www.epicentro.iss.it/en/ coronavirus/sars-cov-2-healthy-lifestyles (accessed on 12 August 2021).

54. López-Sánchez, G.F.; López-Bueno, R.; Gil-Salmerón, A.; Zauder, R.; Skalska, M.; Jastrzębska, J.; Jastrzębski, Z.; Schuch, F.B.; Grabovac, I.; Tully, M.A.; et al. Comparison of physical activity levels in Spanish adults with chronic conditions before and during COVID-19 quarantine. Eur. J. Publ. Health 2021, 31, 161-166. [CrossRef]

55. Ammar, A.; Brach, M.; Trabelsi, K.; Chtourou, H.; Boukhris, O.; Masmoudi, L.; Bouaziz, B.; Bentlage, E.; How, D.; Ahmed, M.; et al. Effects of COVID-19 home confinement on eating behaviour and physical activity: Results of the ECLB-COVID19 International Online Survey. Nutrients 2020, 12, 1583. [CrossRef]

56. López-Bueno, R.; Calatayud, J.; Casaña, J.; Casajús, J.A.; Smith, L.; Tully, M.A.; Andersen, L.L.; López-Sánchez, G.F. COVID-19 Confinement and health risk behaviors in Spain. Front. Psychol. 2020, 11, 1426. [CrossRef] [PubMed]

57. Balanzá-Martínez, V.; Kapczinski, F.; de Azevedo Cardoso, T.; Atienza-Carbonell, B.; Rosa, A.R.; Mota, J.C.; De Boni, R.B. The assessment of lifestyle changes during the COVID-19 pandemic using a multidimensional scale. Rev. Psiquiatr. Salud Ment. 2021, 14, 16-26. [CrossRef] [PubMed]

58. Martínez-de-Quel, Ó.; Suárez-Iglesias, D.; López-Flores, M.; Pérez, C.A. Physical activity, dietary habits and sleep quality before and during COVID-19 lockdown: A longitudinal study. Appetite 2020, 158, 105019. [CrossRef] [PubMed]

59. Ruiz-Roso, M.B.; de Carvalho Padilha, P.; Mantilla-Escalante, D.C.; Ulloa, N.; Brun, P.; Acevedo-Correa, D.; Arantes Ferreira Peres, W.; Martorell, M.; Aires, M.T.; de Oliveira Cardoso, L.; et al. COVID-19 confinement and changes of adolescent's dietary trends in Italy, Spain, Chile, Colombia and Brazil. Nutrients 2020, 12, 1807. [CrossRef]

60. Bourdas, D.I.; Zacharakis, E.D. Evolution of changes in physical activity over lockdown time: Physical activity datasets of four independent adult sample groups corresponding to each of the last four of the six COVID-19 lockdown weeks in Greece. Data Brief 2020, 32, 106301. [CrossRef] [PubMed]

61. Castañeda-Babarro, A.; Arbillaga-Etxarri, A.; Gutiérrez-Santamaría, B.; Coca, A. Physical activity change during COVID-19 confinement. Int. J. Environ. Res. Public Health 2020, 17, 6878. [CrossRef]

62. García-Tascón, M.; Sahelices-Pinto, C.; Mendaña-Cuervo, C.; Magaz-González, A.M. The Impact of the COVID-19 confinement on the habits of PA practice according to gender (male/female): Spanish case. Int. J. Environ. Res. Public Health 2020, $17,6961$. [CrossRef] 
63. Maugeri, G.; Castrogiovanni, P.; Battaglia, G.; Pippi, R.; D’Agata, V.; Palma, A.; Di Rosa, M.; Musumeci, G. The impact of physical activity on psychological health during COVID-19 pandemic in Italy. Heliyon 2020, 6, e04315. [CrossRef]

64. ISS-Epicentro. Nutrition during the COVID-19 Emergency. Available online: https://www.epicentro.iss.it/en/coronavirus/sarscov-2-healthy-lifestyles-nutrition (accessed on 12 August 2021).

65. Sinisterra Loaiza, L.I.; Vázquez Belda, B.; Miranda López, J.M.; Cepeda, A.; Cardelle Cobas, A. Food habits in the Galician population during confinement for COVID-19. Nutr. Hosp. 2020, 37, 1190-1196.

66. Pišot, S.; Milovanović, I.; Šimunič, B.; Gentile, A.; Bosnar, K.; Prot, F.; Bianco, A.; Lo Coco, G.; Bartoluci, S.; Katović, D.; et al. Maintaining everyday life praxis in the time of COVID-19 pandemic measures (ELP-COVID-19 survey). Eur. J. Public Health 2020, 30, 1181-1186. [CrossRef]

67. Di Renzo, L.; Gualtieri, P.; Pivari, F.; Soldati, F.; Attinà, A.; Cinell, G.; Leggeri, C.; Caparello, G.; Barrea, L.; Scerbo, F.; et al. Eating habits and lifestyle changes during COVID-19 lockdown: An Italian survey. J. Transl. Med. 2020, 18, 229. [CrossRef]

68. Pietrobelli, A.; Pecoraro, L.; Ferruzzi, A.; Heo, M.; Faith, M.; Zoller, T.; Antoniazzi, F.; Piacentini, G.; Fearnbach, S.N.; Heymsfield, S.B. Effects of COVID-19 lockdown on lifestyle behaviors in children with obesity living in Verona, Italy: A longitudinal study. Obesity 2020, 28, 1382-1385. [CrossRef]

69. Cancello, R.; Soranna, D.; Zambra, G.; Zambon, A.; Invitti, C. Determinants of the lifestyle changes during COVID-19 pandemic in the residents of northern Italy. Int. J. Environ. Res. Public Health 2020, 17, 6287. [CrossRef] [PubMed]

70. Correia, T.; Dussault, G.; Pontes, C. The impact of the financial crisis on human resources for health policies in three southernEurope countries. Health Policy 2015, 119, 1600-1605. [CrossRef] [PubMed]

71. Rachiotis, G.; Kourousis, C.; Kamilaraki, M.; Symvoulakis, E.K.; Dounias, G.; Hadjichristodoulou, C. Medical supplies shortages and burnout among greek health care workers during economic crisis: A pilot study. Int. J. Med. Sci. 2014, 11, 442-447. [CrossRef] [PubMed] 